Review

\title{
Biochars and Engineered Biochars for Water and Soil Remediation: A Review
}

\author{
Tariqul Islam ${ }^{1,2,3}$ (), Yanliang $\mathrm{Li}^{1}$ and Hefa Cheng ${ }^{2, *}$ (i) \\ 1 School of Environment and Civil Engineering, Dongguan University of Technology, Dongguan 523808, China; \\ tariqul.acee@sau.ac.bd (T.I.); ylli@dgut.edu.cn (Y.L.) \\ 2 College of Urban and Environmental Sciences, Peking University, Beijing 100871, China \\ 3 Department of Agricultural Construction and Environmental Engineering, Sylhet Agricultural University, \\ Sylhet 3100, Bangladesh \\ * Correspondence: hefac@umich.edu; Tel.: +86-10-62761070
}

check for

updates

Citation: Islam, T.; Li, Y.; Cheng, H. Biochars and Engineered Biochars for Water and Soil Remediation: A Review. Sustainability 2021, 13, 9932. https://doi.org/10.3390/su13179932

Academic Editor: Adriano Sofo

Received: 20 July 2021

Accepted: 1 September 2021

Published: 4 September 2021

Publisher's Note: MDPI stays neutral with regard to jurisdictional claims in published maps and institutional affiliations.

Copyright: (c) 2021 by the authors. Licensee MDPI, Basel, Switzerland. This article is an open access article distributed under the terms and conditions of the Creative Commons Attribution (CC BY) license (https:// creativecommons.org/licenses/by/ $4.0 /)$.
Abstract: Biochars (BCs) are considered as ecofriendly and multifunctional materials with significant potential for remediation of contaminated water and soils, while engineered biochars (E-BCs) with enlarged surface areas and abundant surface functional groups can perform even better in environmental remediation. This review systematically summarizes the key physical and chemical properties of BCs that affect their pollutant sorption capacities, major methods employed for modification of E-BCs, the performance of BCs/E-BCs in removing major types of organic (e.g., antibiotics and pesticides) and inorganic pollutants (e.g., heavy metals), and the corresponding removal mechanisms. The physical and chemical properties of BCs-such as ash or mineral contents, aromaticity, surface structures, $\mathrm{pH}$, and surface functional groups (e.g., $\mathrm{C}=\mathrm{O},-\mathrm{COOH},-\mathrm{OH}$, and $-\mathrm{NH}_{2}$ ) - depend primarily on their feedstock sources (i.e., plant, sludge, or fecal) and the pyrolysis temperature. Ion exchange, precipitation, electrostatic attraction, and complexation are the main mechanisms involved in the adsorption of inorganic pollutants on BCs/E-BCs, whereas hydrogen bonding, pore filling, electrostatic attraction, hydrophobic interaction, and van der Waals forces are the major driving forces for the uptake of organic pollutants. Despite their significant promises, more pilot and field scale investigations are necessary to demonstrate the practical applicability and viability of BCs/E-BCs in water and soil remediation.

Keywords: porous carbonaceous material; environmental remediation; adsorption; aromaticity; alkalinity; surface functional group

\section{Introduction}

Pollution of surface water and soils by a wide range of organic pollutants, such as pesticides, antibiotics, and inorganic pollutants-e.g., arsenic (As), lead $(\mathrm{Pb})$, and mercury (Hg) — has become a major environmental problem over the last decades [1,2]. Pollution of surface water and soils threats food security and poses risk to the ecosystem and human health, which has attracted wide attention of the regulatory authority and the general public [3]. As a result, there has been significant need to develop eco-friendly and costeffective technologies to remediate contaminated water and soils [4]. In this endeavor, application of biochars (BCs) and engineered biochars (E-BCs) converted from various types of biomass has emerged as a promising approach.

BCs are porous carbonaceous materials produced from biomass (wood, leaves, manure, and other waste materials) pyrolyzed under elevated temperatures $\left(300-700{ }^{\circ} \mathrm{C}\right)$ with the absence of air or the presence of insufficient air [5]. Waste biomass derived from green sources-such as food, forestry, and agricultural wastes-are often used as feedstocks to produce biochar [6]. After being pyrolyzed, the labile carbon in the feedstocks is converted into more recalcitrant forms in BCs, which can last hundreds of years [7]. Although greenhouse gas emissions occur during the production of BCs, they could still be used as 
an aid to mitigate climate change [7]. With the feedstocks being mostly waste materials, $\mathrm{BC}$ production is a great approach for waste management, and bioenergy (bio-oil/syngas) can also be captured as by-product during the production process [2]. Furthermore, BCs have porous structures, high surface areas, and abundant surface functional groups that are similar to those present on activated carbon, making them excellent sorbents for both organic and inorganic pollutants in contaminated water and soils [8].

$\mathrm{BCs}$ have received significant attention in various fields of research (e.g., agriculture, material science, and environmental science) over the past decade. A recent study proposed that they are even more promising in environmental management applications compared to carbon sequestration and soil improvement [8]. As depicted on Figure 1, the number of publications on the topic of "biochar" has increased by more than 30-fold from 2010 to 2020. Over the same period, the number of publications related to application of BCs in soil remediation and water remediation also grew quickly, with slightly more papers published on the former topic. Overall, a growing body of literature indicates that $\mathrm{BC}$ s hold great promises for the removal of different types of pollutants from contaminated water and soils. Comprehensive understanding on the characteristics of BCs, how are they produced, their physical and chemical properties, as well as the specific mechanisms involved in the removal of organic and inorganic pollutants by BCs and E-BCs is needed for their successful application in remediating targeted pollutants from surface water and soils. This review presents an overview on the feedstock materials, synthesis processes, and functional properties of BCs/E-BCs, and their application in the remediation of contaminated water and soils. Special attention is paid to the mechanistic interactions between BCs/E-BCs with the pollutants in surface water and soils. Previous studies focused either on the properties of BCs and E-BCs and synthesis methods, the factors influencing their properties, or the mechanisms of pollutant adsorption on them. The present work intended to provide a systematic review on the BCs/E-BCs for water and soil remediation. The specific objectives of this review include: (i) introducing the key physical and chemical properties of BCs with their driven factors (pyrolysis temperature, type of feedstocks, etc.); (ii) summarizing the physical, chemical, and biological methods employed for tuning engineered BCs; (iii) discussing the potential of BCs/E-BCs in removing organic and inorganic pollutants from water and soil media; (iv) elucidating the mechanisms involved in the adsorption of organic and inorganic pollutants on BCs/E-BCs; and (v) identifying the current knowledge gap and priorities in future research on $\mathrm{BCs} / \mathrm{E}-\mathrm{BCs}$.

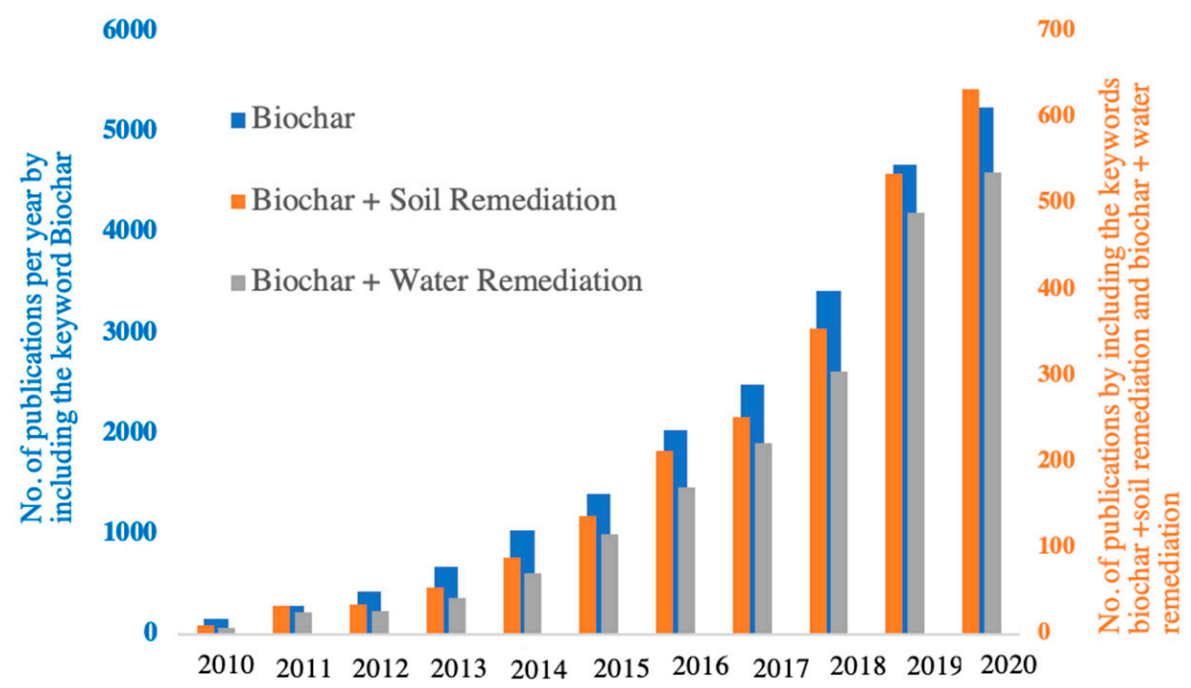

Figure 1. Number of publications in research journals published on topics related to "biochar" between 2010 and 2020. The publications were identified by searching the ISI web of knowledge database (http:/ / apps.webofknowledge.com, accessed on 18 January 2021) with the keywords of "biochar", "biochar + soil remediation", and "biochar + water remediation", respectively. 


\section{Types of BCs and Their Physical and Chemical Properties}

$\mathrm{BC}$ are derived from thermal decomposition of biomass with little oxygen supply, and several major approaches have been developed for their production, including pyrolysis, gasification, and hydrothermal carbonization [9]. The type of feedstock, the production methods, and the pyrolysis temperatures all affect the physical and chemical properties of BCs produced. With distinctly different properties, the type of feedstock plays an important role in the production of BCs [10]. In general, BCs produced from wood materials contain greater amounts of carbon and less nutrients, whereas the manure-based BCs are the opposite, and the characteristics of BCs produced from grasses are in between [11]. Nonetheless, the characteristics of BCs can be altered by the pyrolysis techniques and the pyrolysis temperatures used. In particular, pyrolysis temperature directly influences the chemical and structural properties of BCs, and the nutrient availability in BCs can be drastically changed with increases in the pyrolysis temperature [12]. It has been well observed that the contents of $\mathrm{C}, \mathrm{Ca}, \mathrm{P}, \mathrm{K}$, and ash, as well as the $\mathrm{pH}$ of $\mathrm{BC}$ s increased with the pyrolysis temperature, while their $\mathrm{O}, \mathrm{N}$, and $\mathrm{H}$ contents exhibited the opposite trend [6]. In addition, slow pyrolysis produces $\mathrm{BC}$ s with greater contents of $\mathrm{N}, \mathrm{P}, \mathrm{Ca}$, and $\mathrm{Mg}$, as well as higher cationic exchange capacity (CEC) and surface area, compared to those produced by fast pyrolysis [6].

\subsection{Types of $B C s$}

Several types of carbonaceous materials are related to BCs, but should be clearly differentiated:

Char and charcoal: The term char represents any solid stuffs resulting from the natural or synthetic decomposition of organic substances. Both char and charcoal are produced by pyrolysis in the environment with little or no oxygen [13]. Charcoal is usually used as a fuel for heating, cooking, and other energy-producing purposes, whereas BCs are produced for carbon sequestration and/or as amendment for environmental remediation [14].

Hydrochar: Hydrochar is usually produced as a slurry in water through hydrothermal carbonization. After hydrothermal processing under pressure, the biomass is further used as feedstock to produce BC through pyrolysis, carbonization, or gasification. Hydrochar can be produced from a range of wet waste materials, such as sewage sludge, municipal solid waste, algal residues, aquaculture waste, and animal manure without drying [15].

Agrichar: BC, when used in agricultural applications, is called agrichar or black carbon. It is produced by pyrolyzing the green materials and is applied to farmlands to increase their fertility and productivity. Amendment of agrichar can reduce nutrient leaching in soils, and thus lower the need of chemical fertilizers. Besides minimizing the use of chemical fertilizers and increasing the yields of crops, agrichar can also alleviate the contamination level of soils [13].

\subsection{Modification of $B C s$}

With wide availability of raw materials, tunable physical and chemical properties, $B C$ s have received significant attention in wastewater treatment and carbon sequestration [16]. Nonetheless, the adsorption capacities of raw BCs are often rather low and they are not stable enough for subsequent separation from the treated solutions [17]. To improve the adsorption capacity and stability, E-BCs have been developed by incorporating other materials. The characteristics of nanoparticles can also be imparted into biochar-based nanocomposites (BNCs) [18]. Generally, BNCs are categorized as metal oxide/hydroxide biochar composites, magnetic biochar composites, functional nanoparticle (e.g., graphene oxide, carbon nanotube, chitosan, and nanoscale zerovalent iron) coated biochars [19]. To date, various methods have been developed to prepare modified/engineered BCs. As an example, Figure 2 shows the processes involved in the preparation of magnetic rice husk $\mathrm{BC}$ and its application in removing an organic pollutant from aqueous solution. In this case, impregnation of iron nanoparticles increased the surface area of rice husk BC and significantly improved its adsorption capacity. In addition, the coating by iron nanoparticles 
not only renders the $\mathrm{BC}$ magnetic property, which facilitates its separation from aqueous solution, but also improves its stability [20]. With improved sorption capacities for pollutants and greater stability, such E-BCs are quite promising for application in environmental remediation. The methods that can be used to produce E-BCs from pristine BCs include.

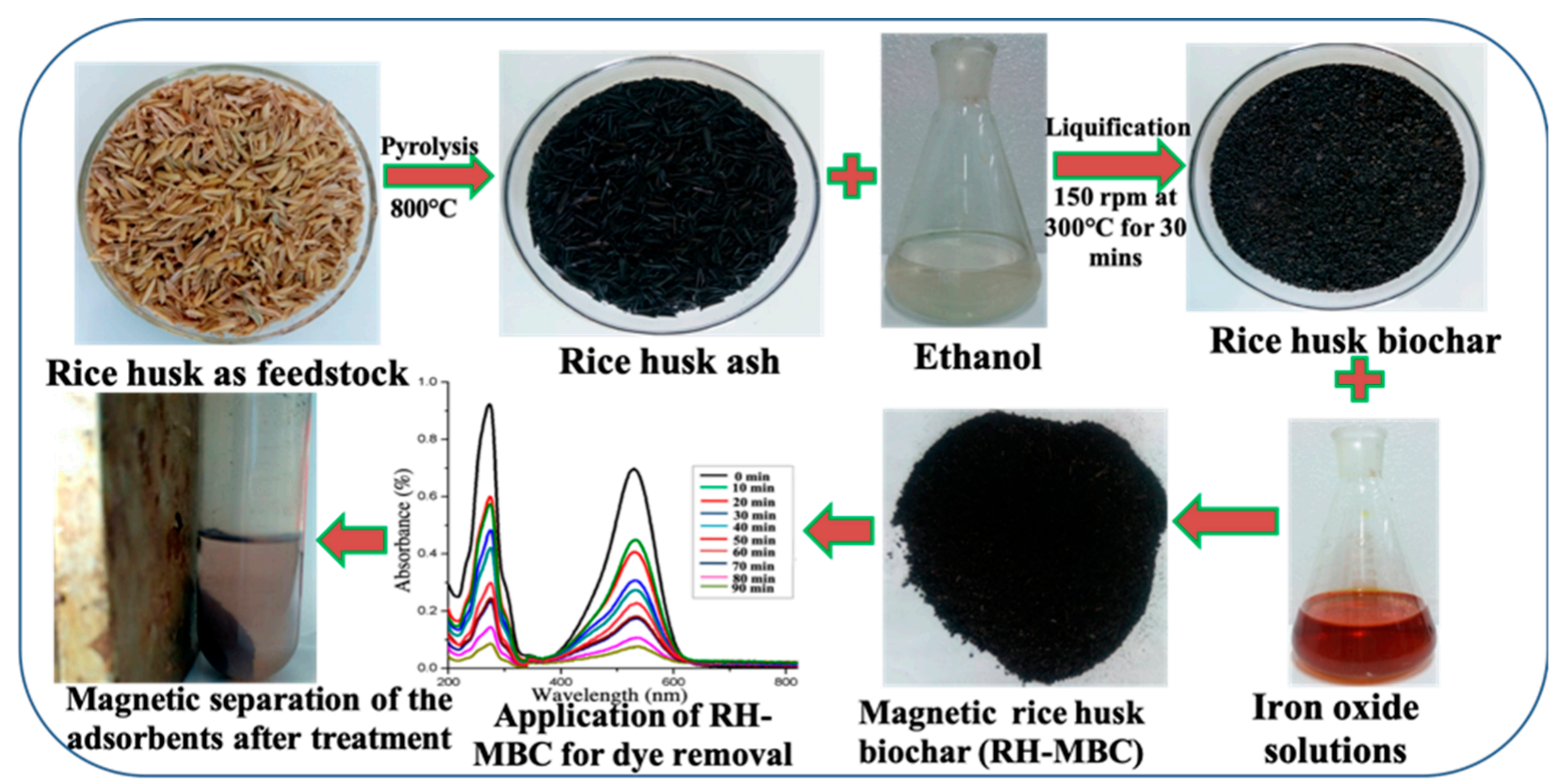

Figure 2. Schematic illustration on the preparation and use of a magnetic rice husk BC.

\subsubsection{Physical Modification}

Modification of BCs using physical methods is low cost and environmental friendly compared to the chemical ones. It improves the physicochemical properties of BCs, such as porosity and permeability, with easy and controllable treatments. The most commonly used techniques in the physical modification of BCs include steam/gas activation, microwave irradiation, ball milling, and magnetization (Figure 3).

Gas/steam activation techniques can increase the surface areas and induce porosity by removing the residues that are trapped inside the porous structures of BCs due to the incomplete combustion. When steam is applied on BCs, they can be activated with hydrogen and carbon dioxide through surface oxidation reactions [21]. As a result, the steam activated E-BCs show greater adsorption capacities towards methane and nitrogen dioxide over the pristine BCs [22].

Microwave irradiation, which rises the temperature of BCs by $200-300{ }^{\circ} \mathrm{C}$ through microwave heating within a short duration, is an emerging technique for modifying BCs. E-BCs prepared from microwave irradiation have greater surface areas compared to those prepared from conventional pyrolysis, and exhibit higher absorption capacities for many pollutants with hydrophilic functional groups [23]. Integration of microwave irradiation with steam activation or impregnation significantly improves the physicochemical properties, such as CEC and water holding capacity, of BCs [24].

Ball milling, in which pristine BCs are broken into powder or nanoparticles, is another common physical modification method. Due to the significant reduction in particle size and significant increase in surface area, the adsorption capacities of BCs are greatly increased [25]. Ball milling can be classified as physical ball milling and chemical ball milling. Physical ball milling is the process where the pristine BCs are grinded into finer particles, whereas chemicals are also added in chemical ball milling for modifying their chemical properties. Particle size and the surface area of BCs are greatly influenced by physical ball milling, whereas chemical ball milling can also modify the functional groups and microporous structure of BCs. The major disadvantages are that the ball milled BCs can 
easily be transported in soils through pores due to their finer sizes, which poses potential risk to groundwater. Moreover, the stability of BCs after pollutant adsorption is also of concern. Magnetic BCs prepared from chemical ball milling can be easily recovered using an external magnetic field. Magnetic BCs have been proven as an efficient adsorbent for organic and inorganic pollutants from water/wastewater [26]. In addition, magnetization process also boosts the catalytic activity and surface charge of BCs, and thus makes them better candidates in environmental remediation.

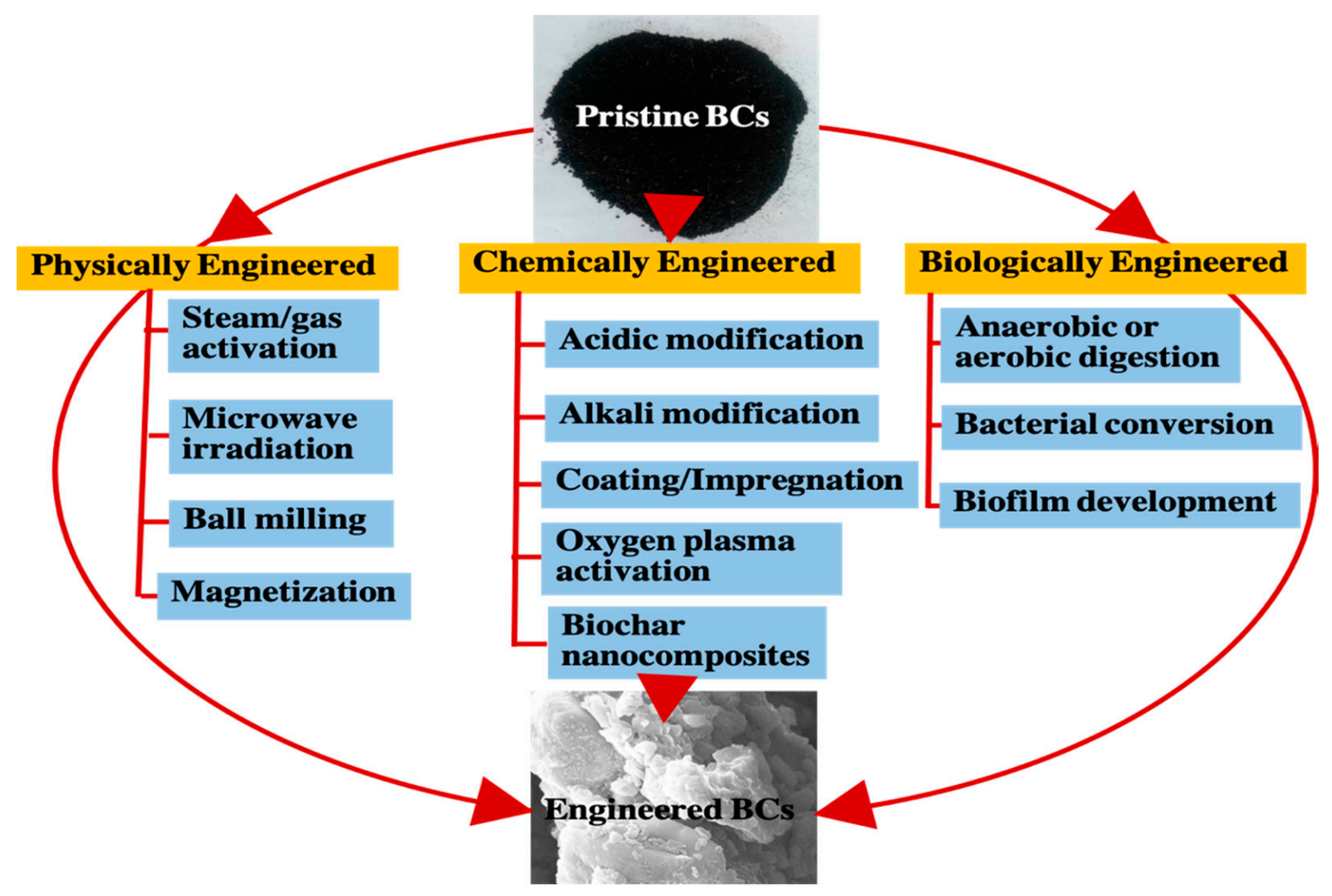

Figure 3. Summary of the methods that can be used to prepare E-BCs from pristine BCs.

\subsubsection{Chemical Modification}

Pristine BCs can be modified through reactions with different chemicals to make them efficient for specific applications by improving their physiochemical characteristics. Chemical modification of BCs can be achieved with a range of processes, such as acidic/alkali modification, coating/impregnation, oxygen plasma activation, and nanocomposite synthesis (Figure 3). BCs modified with acids (e.g., $\mathrm{H}_{2} \mathrm{SO}_{4}, \mathrm{HCl}, \mathrm{HNO}_{3}$, and $\mathrm{H}_{3} \mathrm{PO}_{4}$ ) contain greater amount of oxygen-containing functional groups, while those modified with alkali (e.g., $\mathrm{KOH}$ and $\mathrm{NaOH}$ ) have greater contents of carbon [21]. In addition, treatment with oxidizing agents (e.g., $\mathrm{KMnO}_{4}$ and $\mathrm{Fe}(\mathrm{III})$ ) can significantly increase the pore size and specific surface area of BCs [27].

Recently, oxygen plasma activation has been developed as a novel technique for BC modification, and it bears the advantages of low cost, high efficiency, and environmental friendliness compared to other chemical-based methods [22]. In the modification process, oxygen gas flows through a chamber that has dielectric barrier discharge under a certain pressure. When the reactive gas goes through the plasma chamber, it is converted into many reactive species, including electrons, excited atoms, and oxygen ions [28]. These species react with the functional groups on the surfaces of BCs, which renders the oxygen plasma-activated BCs more active compared to the conventional chemically modified ones.

Chemical modification of pristine BCs by coating or impregnation to form nanocomposites with different metal nanoparticles (NPs) can also increase their functional groups, 
surface area, porosity, and CEC. The impregnation can be achieved through pre-treating feedstock biomass with metal salts, supporting BCs with functional NPs, or forming composites with metal oxide NPs [16]. Pre-treating the biomass with metal salts, clays, or other chemical reagents can enrich the BC with metal hydroxides or nano-metal oxides after pyrolysis [17]. NPs have unique characteristics (e.g., higher surface area and multiple functional groups), which renders them with high affinity for various pollutants. However, instability is a major disadvantage of NPs, which can be simply overcome by immobilizing them on BC matrices. BC-NP composites can have higher porosity and surface areas, multiple functional groups, and good thermal stability, and are proven to be highly efficient in contaminant removal [29].

\subsubsection{Biological Modification}

Biological modification of BCs can be achieved through pre-treating the feedstock biomass with bacterial conversion or anaerobic digestions, and developing biofilms on the external or internal surfaces of BCs (Figure 3). Digestion of waste biomass through anaerobic or aerobic bacteria generates economic value by producing biofuel and biofertilizers. BCs produced from pyrolyzed digestion residues (after anaerobic digestion) have been shown to be highly efficient for removing heavy metals and cationic dyes due to their higher surface areas, high anion exchange capacity, and hydrophobicity [30]. Microorganisms can increase the performance of BCs in pollutant removal by developing biofilms. Biological modified BCs through biofilm are quite promising for removal of pharmaceuticals and other pollutants through immobilization and biotransformation processes [22].

\subsection{Physical and Chemical Properties of BCs}

The physical and chemical properties of BCs depend mostly on the type of biomass used as the feedstock material. They can be classified into three types on the basis of the raw materials used, namely, plant BCs, sludge BCs, and fecal BCs. Out of convenience, BCs are often named directly after their feedstocks, such as bamboo BCs, rice husk BCs, wood BCs, straw BCs, and animal excrement BCs [30]. As a result of the significant difference in the types of feedstocks, as well as the production conditions, the functional properties of BCs can vary largely. Figure 4 schematically illustrates the characteristics of BCs in terms of molecular structure, surface morphology, and elemental composition [20].
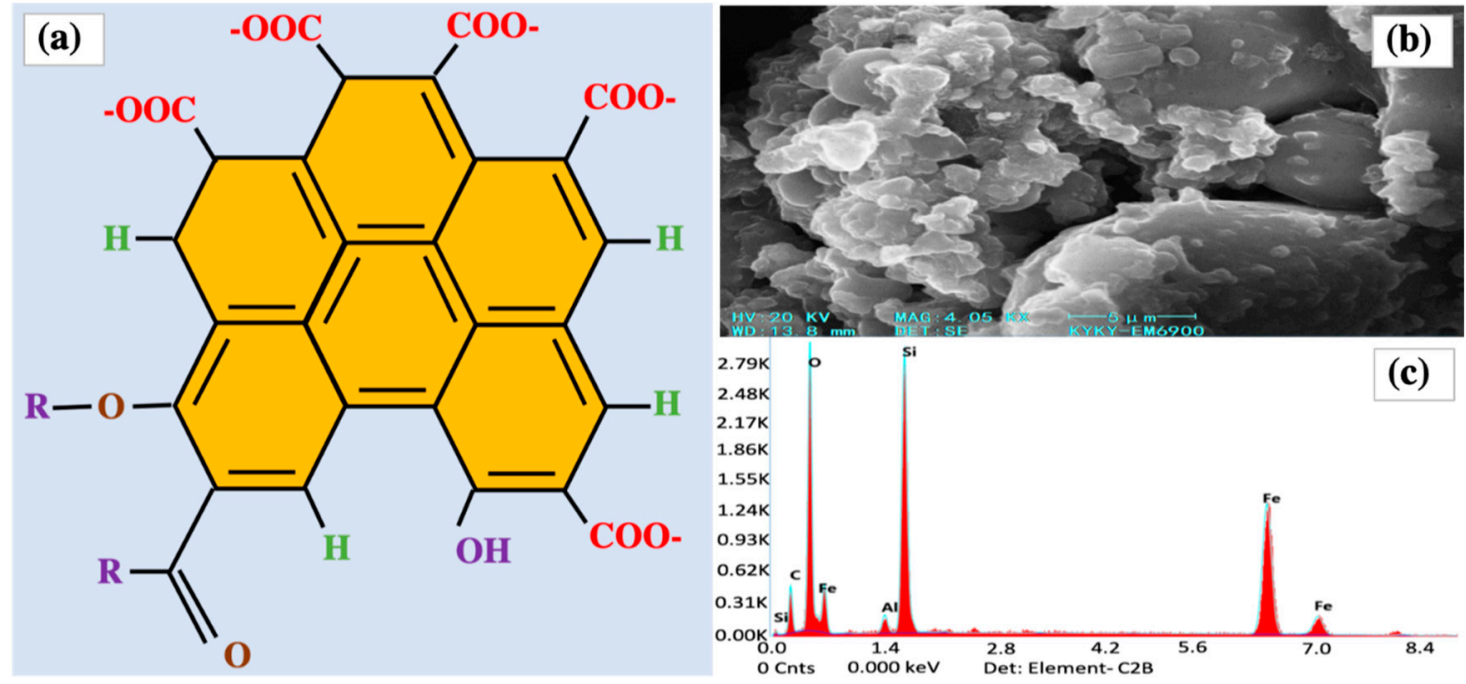

Figure 4. Schematic illustration of the characteristics of BCs: (a) a representative molecular structure; (b) scanning electron microscope image; and (c) elemental composition determined by energy-dispersive X-ray spectroscopy (EDX) [20]. 


\subsubsection{Ash and Mineral Contents}

BCs are mainly composed of carbon (typically around $60 \%$ ), along with other elements, such as hydrogen, oxygen, nitrogen, and sulfur. Carbon exists in BCs in the forms of hydrocarbons, elemental carbon, and carbon with graphite structure [30]. The chemical composition of raw materials and the pyrolysis temperature have major impact on the structure and characteristics of BCs. As shown in Table 1, increasing the pyrolysis temperature reduces the contents of volatile components, as well as the oxygen and nitrogen contents of BCs. During the pyrolysis process, the contents of many elements (e.g., K, $\mathrm{Na}$, $\mathrm{Ca}$, and $\mathrm{Mg}$ ) and nutrients (e.g., $\mathrm{N}$ and $\mathrm{P}$ ) increased in the $\mathrm{BC}$ s produced from animal waste and sludge, which resulted from the gradual removal of the volatile components [30]. Due to the presence of greater amount of inorganic components, the ash contents in animal waste and sludge-derived BCs were higher than those in the plant-based ones, while the former also had a greater number of exchangeable ions and higher CEC.

\subsubsection{Aromaticity and Surface Functional Groups}

The aromaticity of BCs plays an important role in pollutant adsorption as the aromatic structures can function as $\pi$ electron donors or acceptors and form $\pi$ bonds with the pollutants [31]. The ratios of $\mathrm{H} / \mathrm{C}$ and $\mathrm{O} / \mathrm{C}$ of $\mathrm{BC}$ s indicate their aromaticity, which is largely determined by the pyrolysis temperature. Figure 5 shows the van Krevelen diagram for various BCs derived from different sources under different pyrolysis temperatures [32]. Increasing the pyrolysis temperature destroys the alkyl and ester groups, and exposes the aromatic lignin to the $\mathrm{BC}$ surface [32]. With the lignin and cellulose present in plant residue broken down into smaller molecules, the $\mathrm{H} / \mathrm{C}$ and $\mathrm{O} / \mathrm{C}$ ratios in plant-residue $\mathrm{BCs}$ decreased during the process of depolymerization or dehydration. In contrast, animal fecal and sludge BCs did not undergo depolymerization due to the absence of lignocellulosic compounds in their raw materials. As the carbon contents are higher in plant residue BCs, their aromaticity is also higher than in faecal and sludge BCs. The functional groups on $\mathrm{BC}$ surface vary significantly with the pyrolysis temperature, which affects their affinity towards pollutants. In general, BCs produced under higher pyrolysis temperatures have greater affinity towards heavy metals due to their higher aromaticity and specific surface areas. Nonetheless, BCs produced under lower pyrolysis temperatures have more oxygenic functional groups, and thus can still be effective for heavy metal adsorption through complexation [32].

The pyrolysis temperature greatly influences the surface functional groups of $\mathrm{BCs}$. It has been observed that the amount of $-\mathrm{OH}, \mathrm{C}=\mathrm{O}$, and $\mathrm{C}-\mathrm{O}-\mathrm{C}$ functional groups on $\mathrm{BCs}$ decreased with increasing pyrolysis temperature, whereas the $\mathrm{C}=\mathrm{C}$ and $-\mathrm{CH}_{2}$ functional groups could be well preserved in pyrolysis [21]. In general, the acidic functional groups and the yield of BCs both decreased, whereas the alkaline functional groups, $\mathrm{pH}$, and ash contents of BC increased with increases in pyrolysis temperature [33]. Based on the heating rate, the pyrolysis process can be classified as slow pyrolysis and fast pyrolysis. In the slow pyrolysis process, heating occurs over a rather long time (minute to hours), while heating takes place for milliseconds to produce char, biogas, and syngas in the latter. Consequently, BC produced from fast pyrolysis contains greater amount of un-pyrolyzed biomass compared to those produced with slow pyrolysis. Subsequently, BCs produced under these conditions could have significantly different physiochemical properties [33]. 


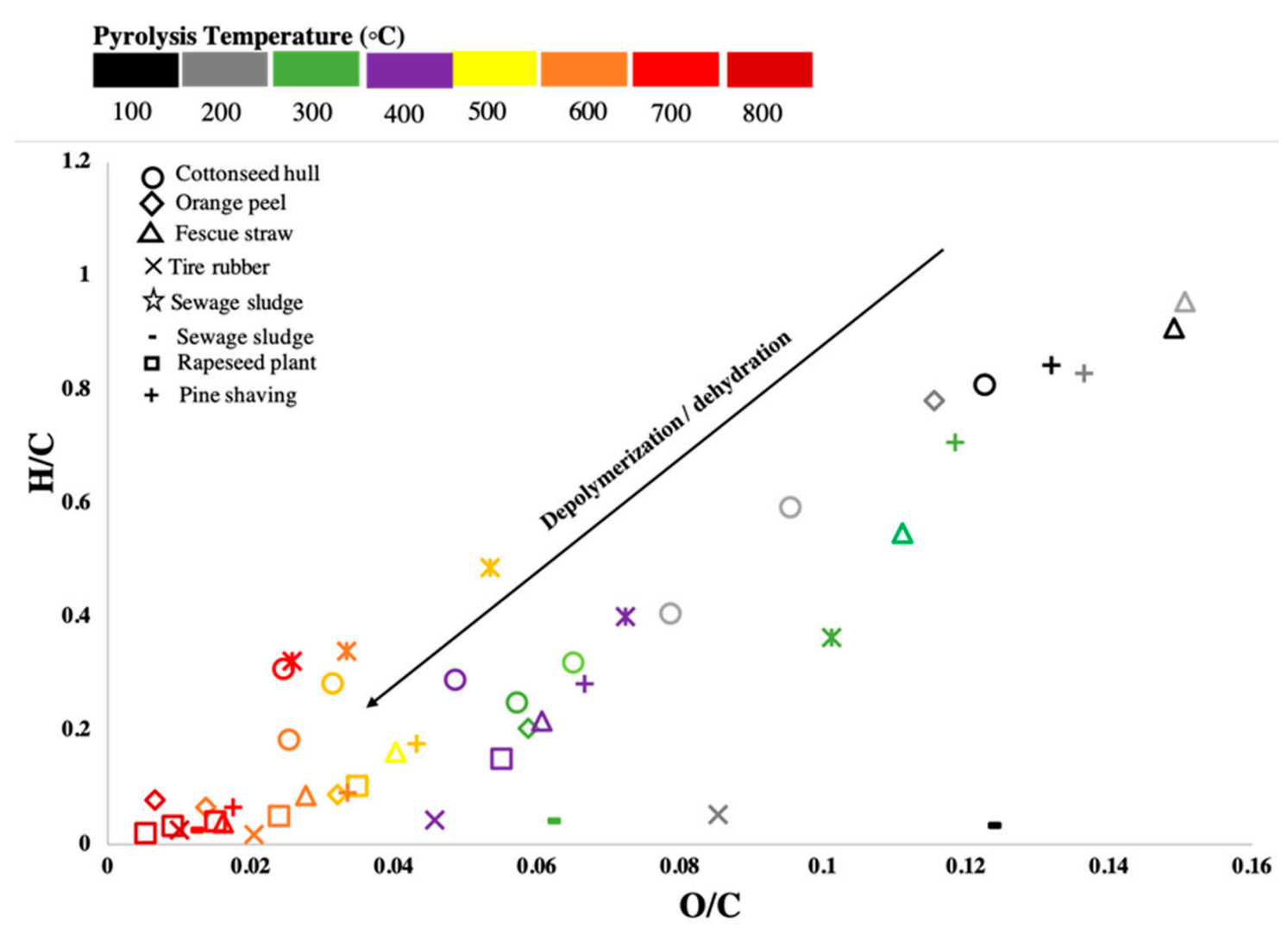

Figure 5. van Krevelen diagram for BCs derived from various sources under different pyrolysis temperatures (data are from those summarized in Table 1). Lower $\mathrm{H} / \mathrm{C}$ and $\mathrm{O} / \mathrm{C}$ ratios are indicative of higher aromaticity of the BC.

\subsubsection{Surface Area and Pore Structure}

The morphology and structure of BCs are closely related to the types of raw materials and the pyrolysis temperature. For BCs produced from the same feedstock, their surface areas typically increase with the pyrolysis temperature [2]. In general, the surface areas of $\mathrm{BCs}$ produced from animal fecal waste and sludge are lower than those derived from plant residues because of their lower carbon contents and lower degree of aromatization. Pyrolysis temperature affects the crystalline structure and pore structure of BCs. Park and co-workers reported that the surface area of sesame straw BCs increased from 6.9 to $289.2 \mathrm{~m}^{2} \cdot \mathrm{g}^{-1}$ while their pore volume increased from 0.0716 to $0.1433 \mathrm{~cm}^{3} \cdot \mathrm{g}^{-1}$ when the pyrolysis temperature was increased from 500 to $600{ }^{\circ} \mathrm{C}$ [9]. During the pyrolysis process of $\mathrm{BCs}$, cleavage of chemical bonds and formation of different functional groups (e.g., - $\mathrm{COOH}$ and -CO) occur in the first stage at temperature $<200{ }^{\circ} \mathrm{C}$, due to the evaporation of moisture and light volatiles. Decomposition of cellulose and hemicellulose occurs in the second stage with temperature ranging from 200 to $500{ }^{\circ} \mathrm{C}$. The strong bonds of lignin and organic matter are broken in the third state at temperatures above $500{ }^{\circ} \mathrm{C}$ [34]. As a result, the physicochemical properties and structure of $\mathrm{BCs}$ are strongly correlated with the pyrolysis temperature. The structure of carbon (C-structure) present in $\mathrm{BC}$ has been observed to respond directly to the change in pyrolysis temperature (Figure 6). BCs produced through pyrolysis in the temperature range of 300 to $400{ }^{\circ} \mathrm{C}$ have amorphous $\mathrm{C}$-structure, while those pyrolyzed between 600 and $700{ }^{\circ} \mathrm{C}$ and above $1200^{\circ} \mathrm{C}$ have turbostatic and graphite structures, respectively [34]. 
Table 1. Summary of selected physical and chemical properties of BCs produced from different sources of feedstock.

\begin{tabular}{|c|c|c|c|c|c|c|c|c|c|c|}
\hline \multirow[b]{2}{*}{$\begin{array}{c}\text { Source } \\
\text { Material }\end{array}$} & \multirow[b]{2}{*}{ Feedstock } & \multirow[b]{2}{*}{$\begin{array}{c}\text { Pyrolysis } \\
\text { Temperature } \\
\left({ }^{\circ} \mathrm{C}\right)\end{array}$} & \multicolumn{7}{|c|}{ BC Properties } & \multirow[b]{2}{*}{ Reference } \\
\hline & & & $\begin{array}{l}\text { Ash } \\
(\%)\end{array}$ & $\mathrm{pH}$ & $\mathrm{C}(\%)$ & H (\%) & $\mathrm{O}(\%)$ & N (\%) & $\begin{array}{c}\text { Pore } \\
\text { Volume } \\
\left(\mathrm{cm}^{3} \cdot \mathrm{g}^{-1}\right)\end{array}$ & \\
\hline \multirow{52}{*}{ Plant } & Fescue straw & 100 & 6.9 & - & 48.60 & 7.25 & 44.10 & 0.64 & 1.8 & {$[35]$} \\
\hline & Pine shaving & 100 & 1.2 & - & 50.60 & 6.68 & 42.70 & 0.05 & 1.6 & [35] \\
\hline & Orange peel & 150 & 0.5 & - & 50.60 & 6.20 & 41.00 & 1.75 & 22.8 & [36] \\
\hline & Pine shaving & 200 & 1.5 & - & 50.90 & 6.95 & 42.20 & 0.04 & 2.3 & [35] \\
\hline & Orange peel & 200 & 0.3 & - & 57.90 & 5.53 & 34.40 & 1.88 & 7.8 & [36] \\
\hline & Cottonseed hull & 200 & 3.1 & - & 51.90 & 6.00 & 40.50 & 0.60 & - & [37] \\
\hline & Fescue straw & 200 & 5.7 & - & 47.20 & 7.11 & 45.10 & 0.61 & 3.3 & [35] \\
\hline & Orange peel & 250 & 1.1 & - & 65.10 & 5.12 & 26.50 & 2.22 & 33.3 & [36] \\
\hline & Pine shaving & 300 & 1.5 & - & 54.80 & 6.50 & 38.70 & 0.05 & 3.0 & [35] \\
\hline & Orange peel & 300 & 1.6 & - & 69.30 & 4.51 & 22.20 & 2.36 & 32.3 & [36] \\
\hline & Buffalo weed & 300 & 20.4 & 8.7 & 78.09 & 4.26 & 7.44 & 10.21 & 4.0 & [5] \\
\hline & Fescue straw & 300 & 9.4 & - & 59.70 & 6.64 & 32.70 & 1.02 & 4.5 & [35] \\
\hline & Peanut shell & 300 & 1.2 & 7.8 & 68.27 & 3.85 & 25.89 & 1.91 & 3.1 & [38] \\
\hline & Soybean stover & 300 & 10.4 & 7.3 & 68.81 & 4.29 & 24.99 & 1.88 & 5.6 & [38] \\
\hline & Cottonseed hull & 350 & 5.7 & - & 77.00 & 4.53 & 15.70 & 1.90 & 4.7 & [37] \\
\hline & Orange peel & 350 & 2.0 & - & 73.20 & 4.19 & 18.30 & 2.30 & 51.0 & [36] \\
\hline & Pine shaving & 400 & 1.1 & - & 74.10 & 4.95 & 20.90 & 0.06 & 28.7 & [35] \\
\hline & Orange peel & 400 & 2.1 & - & 71.70 & 3.48 & 20.80 & 1.92 & 34.0 & [36] \\
\hline & Fescue straw & 400 & 16.3 & - & 77.30 & 4.70 & 16.70 & 1.24 & 8.7 & [35] \\
\hline & Wheat straw & 400 & 9.7 & 9.1 & 65.70 & 4.05 & - & 1.05 & 4.8 & [39] \\
\hline & Rapeseed plant & 400 & 12.2 & - & 71.34 & 3.93 & 10.84 & 1.43 & 16.0 & [40] \\
\hline & Spruce wood & 400 & 1.9 & 6.9 & 63.50 & 5.48 & - & 1.02 & 1.8 & [39] \\
\hline & Soybean stover & 400 & - & - & 44.10 & - & - & 2.38 & - & [41] \\
\hline & Saw dust & 450 & 1.1 & 5.9 & 72.0 & 3.5 & 24.41 & 0.08 & - & [42] \\
\hline & Corn stover & 450 & 58 & - & 33.20 & 1.40 & 8.60 & 0.81 & 12.0 & [43] \\
\hline & Wheat straw & 460 & 12.0 & 8.7 & 72.40 & 3.15 & - & 1.07 & 2.8 & [39] \\
\hline & Spruce wood & 460 & 3.0 & 8.7 & 79.60 & 3.32 & - & 1.24 & 14.2 & [39] \\
\hline & Pine shaving & 500 & 1.4 & - & 81.90 & 3.54 & 14.50 & 0.08 & 196.0 & [35] \\
\hline & Corn stover & 500 & 32.8 & 7.2 & 57.29 & 2.86 & 5.45 & 1.47 & 3.1 & [44] \\
\hline & Cottonseed hull & 500 & 7.9 & - & 87.50 & 2.82 & 7.60 & 1.50 & - & {$[37]$} \\
\hline & Corn cob & 500 & 13.3 & 7.8 & 77.60 & 3.05 & 5.11 & 0.85 & - & [44] \\
\hline & Orange peel & 500 & 4.3 & - & 71.40 & 2.25 & 20.30 & 1.83 & 42.4 & [36] \\
\hline & Fescue straw & 500 & 15.4 & - & 82.20 & 3.32 & 13.40 & 1.09 & 50.0 & [35] \\
\hline & Rapeseed plant & 500 & 12.9 & - & 75.03 & 2.62 & 7.79 & 1.41 & 15.7 & [40] \\
\hline & Wheat straw & 525 & 12.7 & 9.2 & 74.40 & 2.83 & - & 1.04 & 14.2 & [39] \\
\hline & Spruce wood & 525 & 4.7 & 8.6 & 78.30 & 3.04 & - & 1.17 & 40.4 & [39] \\
\hline & Saw dust & 550 & 2.8 & 12.1 & 85.0 & 1.00 & 13.68 & 0.30 & - & [42] \\
\hline & Orange Peel & 600 & 4.1 & - & 77.80 & 1.97 & 14.40 & 1.80 & 7.8 & [36] \\
\hline & Rapeseed plant & 600 & 13.9 & - & 78.48 & 1.88 & 3.94 & 1.53 & 17.6 & [40] \\
\hline & Fescue straw & 600 & 18.9 & - & 89.00 & 2.47 & 7.60 & 0.99 & 75.0 & [35] \\
\hline & Cottonseed hull & 650 & 8.3 & - & 91.00 & 1.26 & 5.90 & 1.60 & 34.0 & [37] \\
\hline & Pine shaving & 700 & 1.7 & - & 92.30 & 1.62 & 6.00 & 0.08 & 347.0 & [35] \\
\hline & Buffalo weed & 700 & 32.3 & 12.3 & 84.96 & 1.09 & 6.56 & 7.40 & 9.3 & [5] \\
\hline & Orange peel & 700 & 2.8 & - & 71.60 & 1.76 & 22.20 & 1.72 & 201.0 & [36] \\
\hline & Fescue straw & 700 & 19.3 & - & 94.20 & 1.53 & 3.60 & 0.70 & 139.0 & [35] \\
\hline & Peanut shell & 700 & 8.9 & 10.6 & 83.76 & 1.75 & 13.34 & 1.14 & 448.2 & [38] \\
\hline & Rapeseed plant & 700 & 14.4 & - & 79.48 & 1.20 & 3.29 & 1.35 & 19.3 & [40] \\
\hline & Soybean stover & 700 & 17.2 & 11.3 & 81.98 & 1.27 & 15.45 & 1.30 & 420.3 & [38] \\
\hline & Pinewood & 700 & 38.8 & 6.6 & 95.30 & 0.82 & 3.76 & 0.12 & 29.0 & {$[45]$} \\
\hline & Cottonseed hull & 800 & 9.2 & - & 90.00 & 0.60 & 7.00 & 1.90 & 322.0 & [37] \\
\hline & Rapeseed plant & 800 & 15.3 & - & 79.51 & 0.72 & 2.61 & 1.45 & 19 & [40] \\
\hline & Rapeseed plant & 900 & - & - & 79.86 & 0.42 & 1.67 & 1.57 & 140.4 & [40] \\
\hline
\end{tabular}


Table 1. Cont.

\begin{tabular}{|c|c|c|c|c|c|c|c|c|c|c|}
\hline \multirow[b]{2}{*}{$\begin{array}{c}\text { Source } \\
\text { Material }\end{array}$} & \multirow[b]{2}{*}{ Feedstock } & \multirow[b]{2}{*}{$\begin{array}{c}\text { Pyrolysis } \\
\text { Temperature } \\
\left({ }^{\circ} \mathrm{C}\right)\end{array}$} & \multicolumn{7}{|c|}{ BC Properties } & \multirow[b]{2}{*}{ Reference } \\
\hline & & & $\begin{array}{l}\text { Ash } \\
(\%)\end{array}$ & $\mathrm{pH}$ & $\mathrm{C}(\%)$ & H (\%) & $\mathrm{O}(\%)$ & N (\%) & $\begin{array}{c}\begin{array}{c}\text { Pore } \\
\text { Volume } \\
\left(\mathrm{cm}^{3} \cdot \mathrm{g}^{-1}\right)\end{array}\end{array}$ & \\
\hline \multirow{20}{*}{$\begin{array}{c}\text { Sludge/ } \\
\text { faecal }\end{array}$} & Paper sludge & 105 & 31.5 & 7.9 & 45.93 & 5.67 & 46.80 & 1.51 & 4.2 & {$[5]$} \\
\hline & Tire rubber & 200 & 15.0 & - & 74.70 & 6.38 & 3.92 & - & - & [46] \\
\hline & Sewage sludge & 300 & 56.6 & 6.8 & 30.72 & 3.11 & 11.16 & 4.11 & 4.5 & [5] \\
\hline & Paper sludge & 300 & 51.2 & 7.8 & 60.00 & 3.71 & 33.81 & 2.49 & 4.3 & [5] \\
\hline & Feedlot residue & 350 & 28.7 & 9.1 & 53.32 & 4.05 & 15.70 & 3.64 & 1.3 & [47] \\
\hline & Turkey litter & 350 & 34.8 & 8.0 & 49.28 & 3.60 & 15.40 & 4.07 & 2.6 & [47] \\
\hline & Poultry litter & 350 & 30.7 & 8.7 & 51.07 & 3.79 & 15.63 & 4.45 & 3.9 & [47] \\
\hline & Tire rubber & 400 & 15.4 & - & 77.70 & 3.56 & 3.34 & - & 24.2 & [46] \\
\hline & Sewage sludge & 400 & 67.1 & 6.6 & 26.62 & 1.93 & 10.67 & 4.07 & 14.1 & [5] \\
\hline & Sewage sludge & 500 & 71.9 & 7.3 & 20.19 & 1.08 & 9.81 & 2.84 & 26.2 & [5] \\
\hline & Sewage sludge & 600 & 74.6 & 8.3 & 24.76 & 0.83 & 8.41 & 2.78 & 35.8 & [5] \\
\hline & Tire rubber & 600 & 15.6 & - & 81.30 & 1.67 & 1.43 & - & 51.5 & [46] \\
\hline & Pine shaving & 600 & 3.7 & - & 89.00 & 2.99 & 8.00 & 0.06 & 392.0 & [35] \\
\hline & Chicken litter & 620 & 53.2 & - & 41.50 & 1.20 & 0.70 & 2.77 & - & [48] \\
\hline & Feedlot residue & 700 & 44.0 & 10.3 & 52.41 & 0.91 & 7.20 & 1.70 & 145.2 & [47] \\
\hline & Sewage sludge & 700 & 76.6 & 8.1 & 22.04 & 0.57 & 7.09 & 1.73 & 54.8 & [5] \\
\hline & Paper sludge & 700 & 73.8 & 9.9 & 59.88 & 0.71 & 37.89 & 1.46 & 145.6 & [5] \\
\hline & Poultry litter & 700 & 46.2 & 10.3 & 45.91 & 1.98 & 10.53 & 2.07 & 50.9 & [47] \\
\hline & Turkey litter & 700 & 49.9 & 9.9 & 44.77 & 0.91 & 5.80 & 1.94 & 66.7 & [47] \\
\hline & Tire rubber & 800 & 10.5 & - & 86.00 & 0.87 & 2.16 & 0.47 & 50.0 & [46] \\
\hline
\end{tabular}

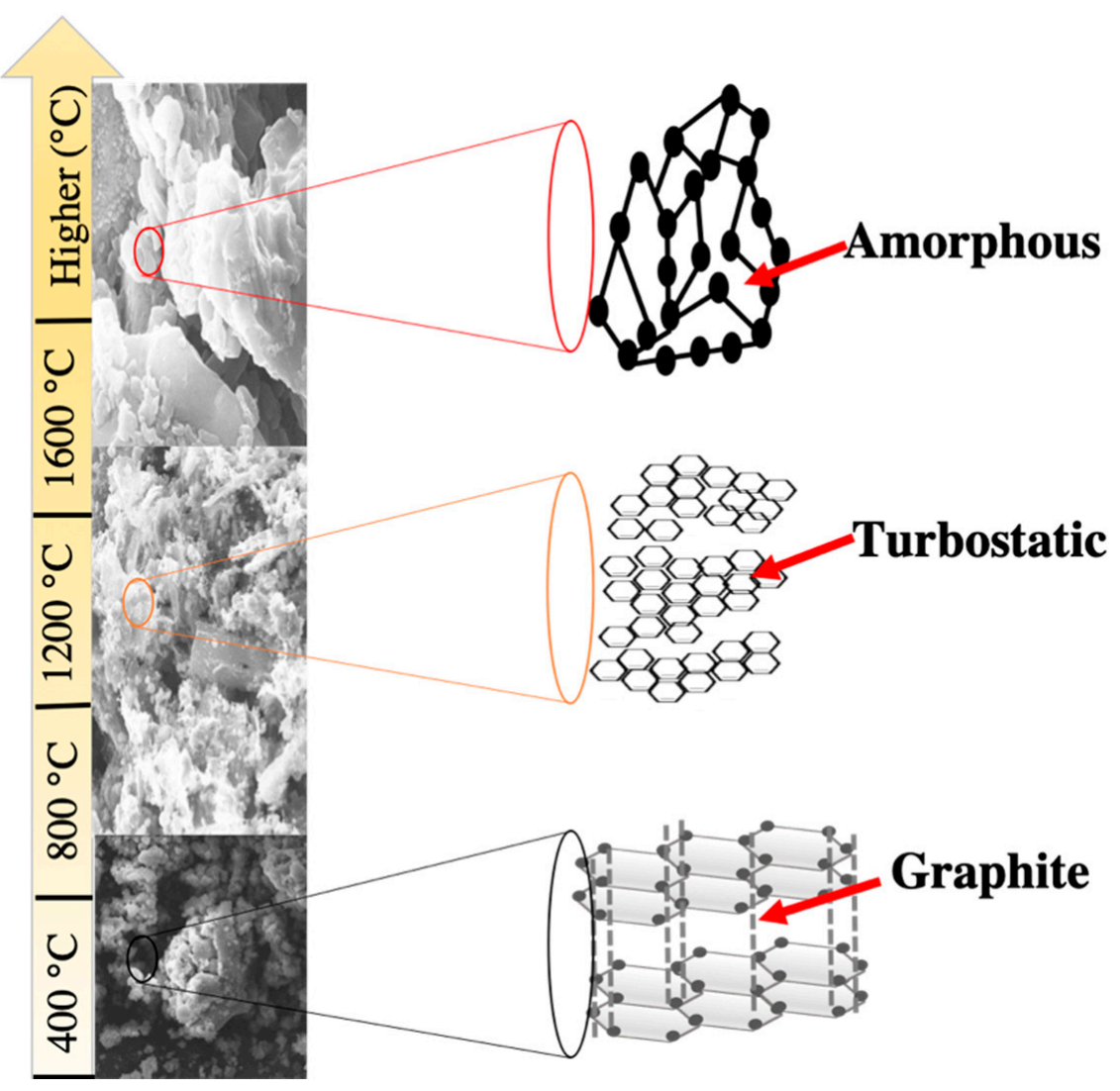

Figure 6. Schematic illustration of the C-structure of BCs produced in different pyrolysis temperature ranges [34]. 


\subsubsection{Alkalinity and $\mathrm{pH}$}

Due to the presence of carbonates and other alkaline components formed during pyrolysis, $\mathrm{BCs}$ are capable of accepting protons without significant changes in their structure. The alkalinity and $\mathrm{pH}$ of $\mathrm{BCs}$ typically increase with the pyrolysis temperature [30]. BCs produced from different feedstocks also vary in alkalinity and $\mathrm{pH}$, with plant-residue $\mathrm{BCs}$ being less alkaline than those produced from animal manure. As a result of their alkalinity, amendment with BCs increases and buffers the soil $\mathrm{pH}$ [5]. In addition, BCs produced under higher pyrolysis temperatures, which are more alkaline, are good sorbents for heavy metals, and they could also promote the formation of metal hydroxide precipitates on their surfaces.

\subsubsection{Hydrologic Properties}

Water repellency of BCs and E-BCs is an important property, which has significant influence on the water retention of soils. The hydraulic properties of soil amended with $\mathrm{BCs}$ can be significantly correlated with the contents of BCs, although these correlations are still not fully understood yet [49]. The hydrologic properties of BCs are directly influenced by the production process/method, type of feedstock, and their particle size and wetting properties [49]. Among these factors, the pyrolysis temperature has the most significant impact on the hydrologic properties of $\mathrm{BCs}$, and thus those of the amended soils (field capacity). It is reported that BCs produced at higher temperatures $\left(400-600{ }^{\circ} \mathrm{C}\right)$ possessed the most desirable hydrologic properties, whereas hydrophobicity is strongly correlated with the presence of aliphatic domains on the surface of BCs produced at lower temperatures [50].

\section{Adsorption of Pollutants on BCs}

Porous carbonaceous materials have been used as efficient sorbents for removing organic and inorganic pollutants from water and soils, with activated carbon being the most widely used one. Activated carbon is essentially charcoal treated with oxygen to increase the surface area and porosity. Both activated carbon and BC are produced through pyrolysis, while the latter is usually not further treated or activated [51]. Moreover, BCs can contain some non-carbonized surface functional groups, such as phenolic, carboxyl, and hydroxyl groups, which could bind effectively with certain pollutants. The multifunctional nature of BCs makes them effective sorbents for both organic and inorganic pollutants [52]. As discussed earlier, the feedstock and pyrolysis temperature largely determine the physical and chemical properties of $\mathrm{BCs}$, and thus their performance in adsorbing pollutants.

\subsection{Adsorption of Organic Pollutants}

Due to their good affinity towards a wide range of organic pollutants and low cost, there has been significant interest in using BCs or E-BCs, instead of activated carbon, to remove organic pollutants from water and soils [53]. Tables 2 and 3 summarize the organic pollutants removed by BCs and E-BCs from water and soils reported in recent studies, respectively. The most widely occurring organic pollutants, including antibiotics, organic dyes, herbicides, pesticides, and polycyclic aromatic hydrocarbons, could all be efficiently removed with BCs and E-BCs through adsorption [20,54-57].

With high specific surface area and microporosity, as well as carbonized surface and a range of functional groups, BCs are good sorbents for organic pollutants [58]. BCs pyrolyzed at low temperatures (below $400{ }^{\circ} \mathrm{C}$ ) have high affinity for organic pollutants due to the partitioning of these compounds into their non-carbonized compartments [31]. Meanwhile, aromaticity and surface polarity are also important properties of BCs that affect the sorption of organic compounds. With increases in the pyrolysis temperature, the surface of BCs becomes more aromatic due to the gradual loss of the oxygen- and hydrogen-containing functional groups [59]. It has been observed that the sorption of deisopropylatrazine on $\mathrm{BCs}$ produced from broiler litter increased with the pyrolysis temperature (up to $700{ }^{\circ} \mathrm{C}$ ) due to the increase in its aromaticity. Nonetheless, an opposite trend was observed in the adsorption of norflurazon and fluridone, with the BC produced 
at $400{ }^{\circ} \mathrm{C}$ exhibiting the highest sorption capacity [21]. This could be explained by the different dominant sorption mechanisms involved with different pollutants. It appears that desisopropylatrazine primarily interacts with the aromatic functional groups of the $\mathrm{BCs}$, while norflurazon and fluridone interact mostly with their polar functional groups. Cationic organic compounds, such as crystal violet dye and norfloxacin, also adsorb on the negatively charged surface of BCs through electrostatic attraction [20,29]. The major mechanisms responsible for the removal of various organic pollutants from water by $\mathrm{BCs}$ are presented in Table 2. While adsorption of organic pollutants on BCs depends primarily on the interactions between them, solution chemistry, such as $\mathrm{pH}$ and ionic strength, can have significant impact through altering the surface charge of the $\mathrm{BCs}$, the speciation of the organic pollutants, and the salting out effect.

Table 2. Summary of organic pollutants removed from water by BCs and the corresponding removal mechanisms involved reported in the literature.

\begin{tabular}{|c|c|c|c|c|c|c|}
\hline $\begin{array}{l}\text { Source } \\
\text { Material }\end{array}$ & $\begin{array}{l}\text { Organic } \\
\text { Pollutant }\end{array}$ & $\begin{array}{l}\text { Feedstock of } \\
\text { BC }\end{array}$ & $\begin{array}{l}\text { Pyrolysis } \\
\text { Temperature } \\
\text { of BC }\left({ }^{\circ} \mathrm{C}\right)\end{array}$ & $\begin{array}{l}\text { Adsorption } \\
\text { Capacity of } \\
\mathrm{BC}\left(\mathrm{mg} \cdot \mathrm{g}^{-1}\right)\end{array}$ & Removal Mechanism & Reference \\
\hline \multirow{19}{*}{ Plant } & Nitrobenzene & Pine needles & $100-700$ & - & Transitional adsorption and partition & {$[60]$} \\
\hline & $\begin{array}{l}\text { Norflurazon } \\
\text { and fluridone }\end{array}$ & $\begin{array}{l}\text { Grass and } \\
\text { wood }\end{array}$ & $200-600$ & - & Sorption on amorphous $\mathrm{C}$ phase & {$[61]$} \\
\hline & Phenanthrene & Soybean stalk & $300-700$ & - & Partitioning & {$[62]$} \\
\hline & Sulfamethazine & Tea waste & $300-700$ & $2.43-7.12$ & $\begin{array}{c}\pi-\pi \text { EDA interaction, cation }-\pi \\
\text { interaction, cation exchange, negative } \\
\text { charge-assisted H-bonding }\end{array}$ & {$[63]$} \\
\hline & Sulfamethazine & $\begin{array}{l}\text { Sicyos angulatus } \\
\text { L. }\end{array}$ & $300-700$ & $15.7-20.6$ & $\begin{array}{l}\pi-\pi \text { EDA, H-bonding, hydrophobic } \\
\text { effect, electrostatic interaction }\end{array}$ & {$[64]$} \\
\hline & Methyl violet & Crop residue & 350 & - & $\begin{array}{l}\text { Electrostatic attraction; interaction } \\
\text { between dye and carboxylate and } \\
\text { phenolic hydroxyl groups; surface } \\
\text { precipitation }\end{array}$ & {$[65]$} \\
\hline & Phenanthrene & Wheat straw & 400 & 6.92 & $\begin{array}{l}\text { H-bonding, hydrophobic effect, } \\
\text { pore-filling, } \pi-\pi \text { interaction }\end{array}$ & {$[61]$} \\
\hline & $\begin{array}{l}\text { Chlorpyrifos } \\
\text { and fipronil }\end{array}$ & Cotton straw & $400-850$ & - & $\begin{array}{l}\text { Adsorption due to high surface area } \\
\text { and microporosity }\end{array}$ & {$[66]$} \\
\hline & Tetracycline & Rice husk & $450-500$ & - & $\begin{array}{l}\text { Formation of } \pi-\pi \text { interactions between } \\
\text { ring structure of tetracycline molecule } \\
\text { and graphite-like sheets of BC }\end{array}$ & {$[42]$} \\
\hline & Norfloxacin & Corn stalk & 500 & 7.25 & $\begin{array}{l}\text { Electrostatic interaction, } \pi-\pi \text { EDA } \\
\text { interaction, pore-filling }\end{array}$ & [29] \\
\hline & Norfloxacin & Reed stalk & 500 & 3.51 & $\begin{array}{l}\text { Electrostatic interaction, } \pi-\pi \text { EDA } \\
\text { interaction, pore-filling }\end{array}$ & [29] \\
\hline & Norfloxacin & Willow branch & 500 & 6.26 & $\begin{array}{l}\text { Electrostatic interaction, } \pi-\pi \text { EDA } \\
\quad \text { interaction, pore-filling }\end{array}$ & [29] \\
\hline & Sulphamethoxazole & Bamboo & 450,600 & - & $\begin{array}{l}\text { Precipitation, ion exchange, cation }-\pi \\
\text { bonding, complexion }\end{array}$ & [67] \\
\hline & Sulphamethoxazole & Pepperwood & 450,600 & - & $\begin{array}{l}\text { Hydrophobic effect, H-bonding, } \pi-\pi \\
\text { EDTA interaction }\end{array}$ & [67] \\
\hline & \multicolumn{2}{|c|}{ Sulphamethoxazole Hickory wood } & 450,600 & - & $\begin{array}{l}\text { Hydrophobic effect, H-bonding, } \pi-\pi \\
\text { EDTA interaction }\end{array}$ & {$[67]$} \\
\hline & Pyrimethanil & $\begin{array}{l}\text { Red gum } \\
\text { woodchip }\end{array}$ & $450-850$ & - & $\begin{array}{l}\text { Adsorption due to high surface area } \\
\text { and microporosity }\end{array}$ & {$[68]$} \\
\hline & Pyrene & Corn stover & 600 & - & Adsorption due to nano-porosity & {$[69]$} \\
\hline & $\begin{array}{l}\text { Crystal violet } \\
\text { dye }\end{array}$ & Rice husk & $600-800$ & 80.04 & $\begin{array}{l}\text { Pore-filling, hydrophobic effect, } \pi-\pi \\
\text { interaction }\end{array}$ & {$[20]$} \\
\hline & $\begin{array}{l}\text { Eriochrome } \\
\text { Black T dye }\end{array}$ & Rice husk & $600-800$ & 5.09 & $\begin{array}{l}\text { Pore-filling, hydrophobic effect, } \pi-\pi \\
\text { interaction }\end{array}$ & {$[20]$} \\
\hline
\end{tabular}


Table 2. Cont.

\begin{tabular}{|c|c|c|c|c|c|c|}
\hline $\begin{array}{l}\text { Source } \\
\text { Material }\end{array}$ & $\begin{array}{l}\text { Organic } \\
\text { Pollutant }\end{array}$ & $\begin{array}{l}\text { Feedstock of } \\
\text { BC }\end{array}$ & $\begin{array}{l}\text { Pyrolysis } \\
\text { Temperature } \\
\text { of BC }\left({ }^{\circ} \mathrm{C}\right)\end{array}$ & $\begin{array}{l}\text { Adsorption } \\
\text { Capacity of } \\
\text { BC }\left(\mathrm{mg}^{-1}\right)\end{array}$ & Removal Mechanism & Reference \\
\hline \multirow{5}{*}{$\begin{array}{l}\text { Sludge/ } \\
\text { faecal }\end{array}$} & Phenanthrene & Poultry litter & $250-400$ & $46.7-61.8$ & $\begin{array}{l}\text { H-bonding, hydrophobic effect, } \\
\text { pore-filling, } \pi-\pi \text { EDA interaction }\end{array}$ & [61] \\
\hline & Atrazine & Dairy manure & 200 & - & Partitioning into organic $\mathrm{C}$ / sorption & [70] \\
\hline & Atrazine & Pig manure & $350-700$ & $0.08-0.32$ & $\begin{array}{l}\text { Pore-filling, hydrophobic effect, } \pi-\pi \\
\text { EDA interaction, H-bonding, partition }\end{array}$ & [71] \\
\hline & Deisopropylatrazine & e Broiler litter & $350-700$ & - & $\begin{array}{l}\text { Sorption due to high surface area and } \\
\text { aromaticity; sorption on non- } \\
\text { carbonized fraction }\end{array}$ & [72] \\
\hline & Carbaryl & Pig manure & $350-700$ & $2.23-2.28$ & $\begin{array}{l}\text { Pore-filling, hydrophobic effect, } \pi-\pi \\
\text { EDA interaction, partition }\end{array}$ & [71] \\
\hline
\end{tabular}

Besides removing organic pollutants from water, $\mathrm{BCs}$ can also be used to adsorb organic pollutants in soils, and thus reduce their bioavailability and risk. Table 3 summarizes the organic pollutants in contaminated soils that could be removed by BCs and the corresponding mechanisms involved. Overall, BCs produced under higher pyrolysis temperatures exhibited higher adsorption capacities for organic pollutants from both water and soils (Tables 2 and 3). This could be attributed to the increases in specific surface areas and microporosity of the BCs produced under higher pyrolysis temperatures. BCs produced from hardwood at pyrolysis temperatures above $400{ }^{\circ} \mathrm{C}$ were found to be effective at suppressing the leaching and biodegradation of simazine in groundwater, which resulted from its effective sorption in the micropores [73,74]. BCs produced from cotton straws at a pyrolysis temperature of $850{ }^{\circ} \mathrm{C}$ could effectively adsorb chlorpyrifos and increased its apparent microbial degradation half-life by 1.6 times [66,75]. As shown in Table 3, BCs produced at lower pyrolysis temperatures are generally less efficient at adsorbing organic pollutants from soils. It is worth noting that the soil matrix is much more complex than that of water, which could complicate the adsorption of organic pollutants. For example, the bioaccessibility of pesticides in soils could be significantly reduced due to coating of the $\mathrm{BC}$ particles by the organic matter present in natural soils [76]. Adsorption of organic pollutants on BCs could also be reduced due to blockage of their micropore spaces.

Table 3. Summary of organic pollutants removed from soils by BCs and the corresponding removal mechanisms involved reported in the literature.

\begin{tabular}{|c|c|c|c|c|c|c|}
\hline $\begin{array}{l}\text { Source } \\
\text { Material }\end{array}$ & Organic Pollutant & BC Feedstock & $\begin{array}{c}\text { Pyrolysis } \\
\text { Temperature } \\
\text { of BC }\left({ }^{\circ} \mathrm{C}\right)\end{array}$ & Performance of BC & Removal Mechanism & Reference \\
\hline \multirow{6}{*}{ Plant } & Fipronil & Cotton straws & 450 & $\begin{array}{c}\text { Decreased plant uptake } \\
\text { by } 20 \%\end{array}$ & $\begin{array}{l}\text { Adsorption, microbial } \\
\text { degradation }\end{array}$ & [66] \\
\hline & Carbamazepine & Oil mallee & 450 & $\begin{array}{l}\text { Decreased plant uptake } \\
\text { by } 32 \%\end{array}$ & Adsorption & [57] \\
\hline & Carbamazepine & wheat chaff & 450 & $\begin{array}{l}\text { Decreased plant uptake } \\
\text { by } 17 \%\end{array}$ & Adsorption & [57] \\
\hline & DEHP & $\begin{array}{l}\text { Bamboo } \\
\text { sawdust }\end{array}$ & 500 & $\begin{array}{l}\text { Decreased plant uptake in } \\
\text { low soil organic } \\
\text { carbon(SOC) soil, but had } \\
\text { no effect for high SOC soil }\end{array}$ & $\begin{array}{l}\text { Adsorption, microbial } \\
\text { degradation }\end{array}$ & [56] \\
\hline & DEHP & Rice straw & 500 & $\begin{array}{l}\text { Decreased plant uptake in } \\
\text { low SOC soil, but had no } \\
\text { effect for high SOC soil }\end{array}$ & $\begin{array}{l}\text { Adsorption, microbial } \\
\text { degradation }\end{array}$ & [56] \\
\hline & $\begin{array}{l}\text { Organochlorine } \\
\text { pesticides }\end{array}$ & Peanut shells & 500 & $\begin{array}{l}\text { Reduced the accessibility } \\
\text { by } 11-75 \%\end{array}$ & $\begin{array}{l}\text { Adsorption, altered soil } \\
\text { microbial community }\end{array}$ & [54] \\
\hline
\end{tabular}


Table 3. Cont.

\begin{tabular}{|c|c|c|c|c|c|c|}
\hline $\begin{array}{l}\text { Source } \\
\text { Material }\end{array}$ & Organic Pollutant & BC Feedstock & $\begin{array}{c}\text { Pyrolysis } \\
\text { Temperature } \\
\text { of BC }\left({ }^{\circ} \mathrm{C}\right)\end{array}$ & Performance of BC & Removal Mechanism & Reference \\
\hline & $\begin{array}{l}\text { Organochlorine } \\
\text { pesticides }\end{array}$ & Rice straw & 500 & $\begin{array}{l}\text { Reduced the accessibility } \\
\text { by } 6-67 \%\end{array}$ & $\begin{array}{l}\text { Adsorption, altered soil } \\
\text { microbial community }\end{array}$ & [54] \\
\hline & $\begin{array}{l}\text { Organochlorine } \\
\text { pesticides }\end{array}$ & Soybean straw & 500 & $\begin{array}{l}\text { Reduced the accessibility } \\
\text { by } 14-86 \%\end{array}$ & $\begin{array}{l}\text { Adsorption, altered soil } \\
\text { microbial community }\end{array}$ & [54] \\
\hline & Carbamazepine & Blue mallee & 520 & $\begin{array}{l}\text { Decreased plant uptake } \\
\text { by } 42 \%\end{array}$ & Adsorption & [57] \\
\hline & Simazine & Hardwood & 450,600 & - & $\begin{array}{l}\text { Sorption due to } \\
\text { abundance of micropores }\end{array}$ & [74] \\
\hline & Pentachlorophenol & Bamboo & 600 & - & $\begin{array}{l}\text { Precipitation, ion } \\
\text { exchange, cation- } \pi \\
\text { bonding, complexion }\end{array}$ & [77] \\
\hline & Phenanthrene & Sawdust & 700 & $2.1-3.4 \times 104 \mathrm{~L} \cdot \mathrm{kg}^{-1}$ & Adsorption & [78] \\
\hline & Aroclors 1254,1260 & Wood waste & 700 & $\begin{array}{l}\text { Reduced concentrations } \\
\text { up to } 58-77 \%\end{array}$ & Adsorption & [79] \\
\hline & $\begin{array}{l}\text { Aroclor } 1242 \text { and } 4 \\
\text { PCB congeners }\end{array}$ & Bamboo & 820 & $\begin{array}{l}\text { Enhanced PCB } \\
\text { degradation }\end{array}$ & $\begin{array}{l}\text { Precipitation, ion } \\
\text { exchange, cation- } \pi \\
\text { bonding, complexion }\end{array}$ & [55] \\
\hline & Chlorpyrifos & Cotton straws & 850 & $\begin{array}{c}\text { Increased half-life of } \\
\text { chlorpyrifos by } 161 \% ; \\
\text { decreased plant uptake by } \\
81 \%\end{array}$ & $\begin{array}{l}\text { Adsorption, microbial } \\
\text { degradation }\end{array}$ & [66] \\
\hline & $\begin{array}{l}\text { Polycyclic aromatic } \\
\text { hydrocarbons } \\
\text { (PAHs) }\end{array}$ & Hard wood & & - & $\begin{array}{l}\text { Sorption and } \\
\text { biodegradation }\end{array}$ & [80] \\
\hline \multirow{4}{*}{$\begin{array}{l}\text { Sludge/ } \\
\text { faecal }\end{array}$} & $\begin{array}{l}\text { Agro chemicals } \\
\text { Atrazine }\end{array}$ & Dairy manure & 450 & - & Sorption & [81] \\
\hline & $\begin{array}{l}\text { Organochlorine } \\
\text { pesticides }\end{array}$ & Sewage sludge & 500 & $\begin{array}{l}\text { Reduced the accessibility } \\
\text { by } 8-69 \%\end{array}$ & $\begin{array}{l}\text { Adsorption, altered soil } \\
\text { microbial community }\end{array}$ & [54] \\
\hline & Diuron & Poultry litter & 550 & $\begin{array}{l}\text { Increased sorption of } \\
\text { diuron on soil by } 5 \text { times }\end{array}$ & Adsorption & [82] \\
\hline & Diuron & $\begin{array}{l}\text { Paper mill } \\
\text { sludge }\end{array}$ & 550 & $\begin{array}{l}\text { Increased sorption of } \\
\text { diuron on soil by } 2 \text { times }\end{array}$ & Adsorption & [82] \\
\hline
\end{tabular}

\subsection{Adsorption of Inorganic Pollutants in Water and Soils}

$\mathrm{BCs}$ are promising sorbents for a range of inorganic pollutants due to their diverse surface characteristics. Heavy metals are the most common inorganic pollutants of significant concerns found in surface water and soils. They can come from a wide range of anthropogenic sources, such as mining and smelting operations, industrial production, power plants, fertilizers, sewage sludge, and animal manure [83-85]. Unlike organic pollutants, heavy metals are not biodegradable and can be easily uptaken by plants, and subsequently transferred to animals and humans through the food chain [5]. BCs produced from different sources can stabilize heavy metals through adsorption [2]. It has been observed that pinewood BCs produced at higher pyrolysis temperatures had greater adsorption capacities towards uranium than those produced at lower temperatures [86], which could be attributed to the increases in the pore space and surface area of the BCs with greater degree of carbonization. The sorption capacities of BCs towards different heavy metals could vary significantly due to the difference in sorption mechanisms and valence of the metal ions.

BCs have significant sorption capacities for heavy metals, and different binding mechanisms could be involved, depending on the types of BCs and solution matrix [6]. Table 4 summarizes the removal of heavy metals from water by BCs and the major mechanisms as reported in recent investigations. Common mechanisms involved in the uptake of 
heavy metals from water by BCs are physical adsorption, surface precipitation, electrostatic outer-sphere complexation, inner-sphere complexation, cation- $\pi$ bonding, and surface complexation $[8,29,83,85]$. More details on the interaction mechanisms between BCs and heavy metals are discussed later. Besides adsorption, BCs also have redox-active oxygencontaining functional groups (e.g., quinone/hydroquinone groups), which could promote redox transformation of the sorbed metal species. For example, BCs could efficiently adsorb $\mathrm{Cr}(\mathrm{VI})$ from water, while they are also capable of converting $\mathrm{Cr}(\mathrm{VI})$ into less toxic $\mathrm{Cr}(\mathrm{III})$, which significantly reduces the risk of pollution [5].

Table 4. Summary of heavy metals removed from water by BCs and the corresponding removal mechanisms reported in the literature.

\begin{tabular}{|c|c|c|c|c|c|c|}
\hline $\begin{array}{l}\text { Source } \\
\text { Material }\end{array}$ & $\begin{array}{l}\text { Heavy } \\
\text { Metal }\end{array}$ & Feedstock of BC & $\begin{array}{l}\text { Pyrolysis } \\
\text { Tempera- } \\
\text { ture of BC } \\
\left({ }^{\circ} \mathrm{C}\right)\end{array}$ & $\begin{array}{l}\text { Removal } \\
\text { Capacity } \\
\left(\mathrm{mg} \cdot \mathrm{g}^{-1}\right)\end{array}$ & Removal Mechanism & Reference \\
\hline \multirow{10}{*}{ Plant } & $\mathrm{Cr}$ & Sugar beat tailing & 300 & - & $\begin{array}{l}\text { Electrostatic attraction, } \\
\text { reduction, complexation }\end{array}$ & [5] \\
\hline & $\mathrm{Pb}$ & Rice husk & 300 & 2.40 & Complexation & [87] \\
\hline & $\mathrm{Cr}$ & Oak wood & $400-450$ & - & $\begin{array}{l}\text { Transitional adsorption } \\
\text { and partition }\end{array}$ & [88] \\
\hline & $\mathrm{Cd}$ & Oak bark & 450 & 7.40 & $\begin{array}{l}\text { Complexation, ion } \\
\text { exchange, precipitation }\end{array}$ & [83] \\
\hline & $\mathrm{Hg}$ & Corn straw & 500 & 4.26 & Complexation & [84] \\
\hline & $\mathrm{Ni}$ & Wheat straw & 600 & 17.7 & $\begin{array}{l}\text { Physical adsorption, } \\
\text { surface precipitation }\end{array}$ & [9] \\
\hline & $\mathrm{Cd}$ & Cauliflower leaves & 600 & 73.8 & $\begin{array}{c}\text { Complexation, ion } \\
\text { exchange, precipitation }\end{array}$ & [83] \\
\hline & $\mathrm{Pb}$ & Banana peels & 600 & 247 & $\begin{array}{c}\text { Complexation, ion } \\
\text { exchange, precipitation }\end{array}$ & [83] \\
\hline & $\mathrm{Pb}$ & Cauliflower leaves & 600 & 178 & $\begin{array}{c}\text { Complexation, ion } \\
\text { exchange, precipitation }\end{array}$ & [83] \\
\hline & $\mathrm{Pb}$ & Cauliflower leaves & 600 & 178 & $\begin{array}{l}\text { Complexation, ion } \\
\text { exchange, precipitation }\end{array}$ & [83] \\
\hline \multirow{7}{*}{$\begin{array}{l}\text { Sludge/ } \\
\text { faecal }\end{array}$} & $\mathrm{Pb}$ & Dairy manure & 200 & - & $\begin{array}{l}\text { Precipitation with } \\
\text { phosphate }\end{array}$ & [70] \\
\hline & $\mathrm{Zn}$ & Dairy manure & $200-350$ & 31.6 & $\begin{array}{l}\text { Surface precipitation, } \\
\text { complexation, cation }-\pi \\
\text { bonding }\end{array}$ & [89] \\
\hline & $\mathrm{Zn}$ & Dairy manure & 350 & 32.8 & $\begin{array}{l}\text { Surface precipitation, } \\
\text { complexation, cation }-\pi \\
\text { bonding }\end{array}$ & [89] \\
\hline & $\mathrm{Cu}$ & Dairy manure & 350 & 54.4 & $\begin{array}{l}\text { Surface precipitation, } \\
\text { complexation, cation }-\pi \\
\text { bonding }\end{array}$ & [89] \\
\hline & $\mathrm{Hg}$ & Sewage sludge & 400 & 594 & Complexation & [29] \\
\hline & $\mathrm{Ni}$ & $\begin{array}{l}\text { Residues of biogas } \\
\text { production }\end{array}$ & 400 & 27.9 & $\begin{array}{l}\text { Physical adsorption, } \\
\text { surface precipitation }\end{array}$ & [9] \\
\hline & $\mathrm{Ni}$ & $\begin{array}{l}\text { Residues of biogas } \\
\text { production }\end{array}$ & 600 & 34.8 & $\begin{array}{l}\text { Physical adsorption, } \\
\text { surface precipitation }\end{array}$ & [9] \\
\hline
\end{tabular}

There have been significant interests in applying BCs for in situ stabilization of heavy metals in agricultural soils to reduce their bioavailability. Table 5 summarizes the heavy 
metals in contaminated soils that were removed by BCs and the corresponding removal mechanisms as reported in the literature. $\mathrm{BCs}$ produced from a range of materials exhibited strong sorption capacities towards heavy metals-including $\mathrm{As}, \mathrm{Cu}, \mathrm{Cd}, \mathrm{Ni}, \mathrm{Zn}$, and $\mathrm{Pb}$-in soils $[2,21,90-93]$. The alkalinity of BCs, which increases the $\mathrm{pH}$ of the amended soil, plays an important role in the immobilization of heavy metals. It is worth noting that some BCs could be enriched with certain heavy metals, depending on the feedstock. For example, Namgay and co-workers reported that the BC produced from Sydney blue gum increased the content of As in the amended soil [94]. With the complicated compositions of BCs and soils, the interactions between heavy metals and the BC-amended soil are still not fully understood. For example, it has been observed that application of wood derived BCs immobilized $\mathrm{Cd}$ and $\mathrm{Zn}$ in the soil, but mobilized $\mathrm{Cu}$ and As $[5,80]$.

Table 5. Summary of heavy metals removed from soils by BCs and the corresponding removal mechanisms reported in the literature.

\begin{tabular}{|c|c|c|c|c|c|c|}
\hline $\begin{array}{l}\text { Source } \\
\text { Material }\end{array}$ & $\begin{array}{l}\text { Heavy } \\
\text { Metal }\end{array}$ & $\begin{array}{l}\text { Feedstock of } \\
\text { BC }\end{array}$ & $\begin{array}{c}\text { Pyrolysis } \\
\text { Temperature } \\
\text { of BC }\left({ }^{\circ} \mathrm{C}\right)\end{array}$ & Performance of BC & Removal Mechanism & Reference \\
\hline \multirow{19}{*}{ Plant } & $\mathrm{Ni}$ & $\begin{array}{l}\text { Licorice root } \\
\text { pulp }\end{array}$ & 350 & $\begin{array}{l}\text { Decreased Ni uptake by plant by } \\
67.2 \%\end{array}$ & Liming effect & {$[91]$} \\
\hline & $\mathrm{Pb}$ & Canola straw & 400 & $\begin{array}{l}\text { Enhanced the adsorption } \\
\text { capacity for } \mathrm{Pb} \text { by } 44 \%\end{array}$ & Adsorption, liming effect & {$[95]$} \\
\hline & $\mathrm{Pb}$ & $\begin{array}{l}\text { Soybean } \\
\text { straw }\end{array}$ & 400 & $\begin{array}{l}\text { Enhanced the adsorption } \\
\text { capacity for } \mathrm{Pb} \text { by } 37.3 \%\end{array}$ & Adsorption, liming effect & [95] \\
\hline & As & Hard wood & 400 & - & Mobilization due to enhanced pH & {$[96]$} \\
\hline & $\mathrm{Pb}$ & $\begin{array}{l}\text { Sugarcane } \\
\text { bagasse }\end{array}$ & 450 & $\begin{array}{l}\text { Decreased Cd bioavailability by } \\
\qquad 5.3-8.4 \%\end{array}$ & Adsorption, precipitation & [92] \\
\hline & $\mathrm{Cu}$ & $\begin{array}{l}\text { Sugarcane } \\
\text { bagasse }\end{array}$ & 450 & $\begin{array}{l}\text { Decreased bioavailability of } \mathrm{Cu} \\
\text { by } 3.4-6.0 \%\end{array}$ & Adsorption & {$[92]$} \\
\hline & $\mathrm{Cd}$ & $\begin{array}{l}\text { Sugarcane } \\
\text { bagasse }\end{array}$ & 450 & $\begin{array}{l}\text { Decreased bioavailability of Cd } \\
\text { by } 2.7-5.6 \%\end{array}$ & Adsorption & [92] \\
\hline & $\mathrm{Cd}$ & Bamboo & 500 & $\begin{array}{c}\text { Decreased exchangeable Cd in } \\
\text { soil by } 12.5 \%\end{array}$ & Adsorption & {$[90]$} \\
\hline & $\mathrm{Cu}$ & Rice straw & 500 & $\begin{array}{l}\text { Reduced } \mathrm{Cu} \text { uptake in roots by } \\
\qquad 35 \%\end{array}$ & Adsorption & {$[2]$} \\
\hline & $\mathrm{Cd}$ & $\begin{array}{l}\text { S-modified } \\
\text { bamboo }\end{array}$ & 500 & $\begin{array}{l}\text { Decreased exchangeable Cd in } \\
\text { soil by } 29.7 \%\end{array}$ & Adsorption & {$[90]$} \\
\hline & $\mathrm{Pb}$ & Walnut shell & 500 & Decreased TCLP-extractable Pb & Adsorption & [87] \\
\hline & $\mathrm{Pb}$ & Bamboo & 500 & Decreased TCLP-extractable Pb & Adsorption & [87] \\
\hline & As & $\begin{array}{l}\text { Activated } \\
\text { wood }\end{array}$ & 550 & $\begin{array}{l}\text { Decrease the concentration of As } \\
\text { in maize shoots significantly }\end{array}$ & Adsorption & [94] \\
\hline & As & $\begin{array}{l}\text { Sydney blue } \\
\text { gum }\end{array}$ & 550 & $\begin{array}{l}\text { Increased the concentration of } \\
\text { available As in soil }\end{array}$ & - & {$[94]$} \\
\hline & $\mathrm{Ni}$ & Rice husk & 550 & $\begin{array}{l}\text { Decreased Ni uptake by plant by } \\
65.2 \%\end{array}$ & Liming effect & [91] \\
\hline & As, $\mathrm{Cu}$ & Hard wood & - & - & $\begin{array}{l}\text { Immobilization of arsenic, } \\
\text { chromium, cobalt, nickel and lead } \\
\text { due to rise in soil pH; mobilization } \\
\text { of copper, zinc, and cadmium due } \\
\text { to high available concentrations in } \\
\text { biochar }\end{array}$ & {$[5]$} \\
\hline & $\mathrm{Zn}$ & $\begin{array}{l}\text { Sydney blue } \\
\text { gum }\end{array}$ & 550 & No effect on Zn uptake by plant & - & [93] \\
\hline & $\mathrm{Ni}$ & Hardwood & 600 & Reduced by 83-98\% & Adsorption & [93] \\
\hline & $\mathrm{Zn}$ & Hardwood & 600 & $\begin{array}{l}\text { Reduced extracted } \mathrm{Zn} \text { by } \\
\qquad 83-98 \%\end{array}$ & Adsorption & [93] \\
\hline
\end{tabular}


Table 5. Cont.

\begin{tabular}{|c|c|c|c|c|c|c|}
\hline $\begin{array}{l}\text { Source } \\
\text { Material }\end{array}$ & $\begin{array}{l}\text { Heavy } \\
\text { Metal }\end{array}$ & $\begin{array}{l}\text { Feedstock of } \\
\text { BC }\end{array}$ & $\begin{array}{c}\text { Pyrolysis } \\
\text { Temperature } \\
\text { of BC }\left({ }^{\circ} \mathrm{C}\right)\end{array}$ & Performance of BC & Removal Mechanism & Reference \\
\hline \multirow{3}{*}{$\begin{array}{l}\text { Sludge/ } \\
\text { faecal }\end{array}$} & $\mathrm{Ni}$ & $\begin{array}{l}\text { Broiler litter } \\
\text { manure }\end{array}$ & 350 & Enhanced immobilization & Adsorption and precipitation & [72] \\
\hline & $\mathrm{Cd}$ & $\begin{array}{l}\text { Broiler litter } \\
\text { manure }\end{array}$ & 350 & Enhanced Cd immobilization & Adsorption, liming effect & [72] \\
\hline & $\begin{array}{l}\mathrm{As}, \mathrm{Cd}, \\
\mathrm{Cr}, \mathrm{Co}, \\
\mathrm{Cu}, \mathrm{Ni}, \\
\mathrm{Pb}, \mathrm{Zn}\end{array}$ & $\begin{array}{l}\text { Sewage } \\
\text { sludge }\end{array}$ & $500-550$ & - & $\begin{array}{l}\text { Immobilization due to enhanced } \\
\qquad \mathrm{pH}\end{array}$ & [5] \\
\hline
\end{tabular}

\subsection{Mechanisms of Pollutant Removal by BCs}

While the physical and chemical properties of organic pollutants are quite different from those of inorganic ones, most of the surface sites and functional groups present on BCs can interact with both types of pollutants. As a result, the sorption mechanisms involved in the adsorption of both organic and inorganic pollutants are discussed together to avoid redundancy. The major mechanisms involved in the adsorption of organic and inorganic pollutants on $\mathrm{BC}$ particles are schematically depicted on parts $\mathrm{a}$ and $\mathrm{b}$ of Figure 7 , respectively. Organic pollutants adsorb onto BCs through a range of mechanisms, including hydrogen bonding, miocropore filling, electrostatic attraction, hydrophobic interaction, and van der Waals forces. Carbonyl $(\mathrm{C}=\mathrm{O})$, carboxyl $(-\mathrm{COOH})$, hydroxyl $(-\mathrm{OH})$, and amino $\left(-\mathrm{NH}_{2}\right)$ functional groups are the main sites for the adsorption of organic pollutants on the BC surface, and they are typically more abundant on plant-based BCs pyrolyzed at relatively low temperatures. The adsorption of inorganic pollutants (primarily heavy metal cations) on BCs occurs primarily through ion exchange, precipitation/co-precipitation, electrostatic attraction, and complexion. In addition, surface precipitation (in the forms of hydroxides, phosphates, and carbonates) could occur for metal cations on the BCs with high alkalinity.

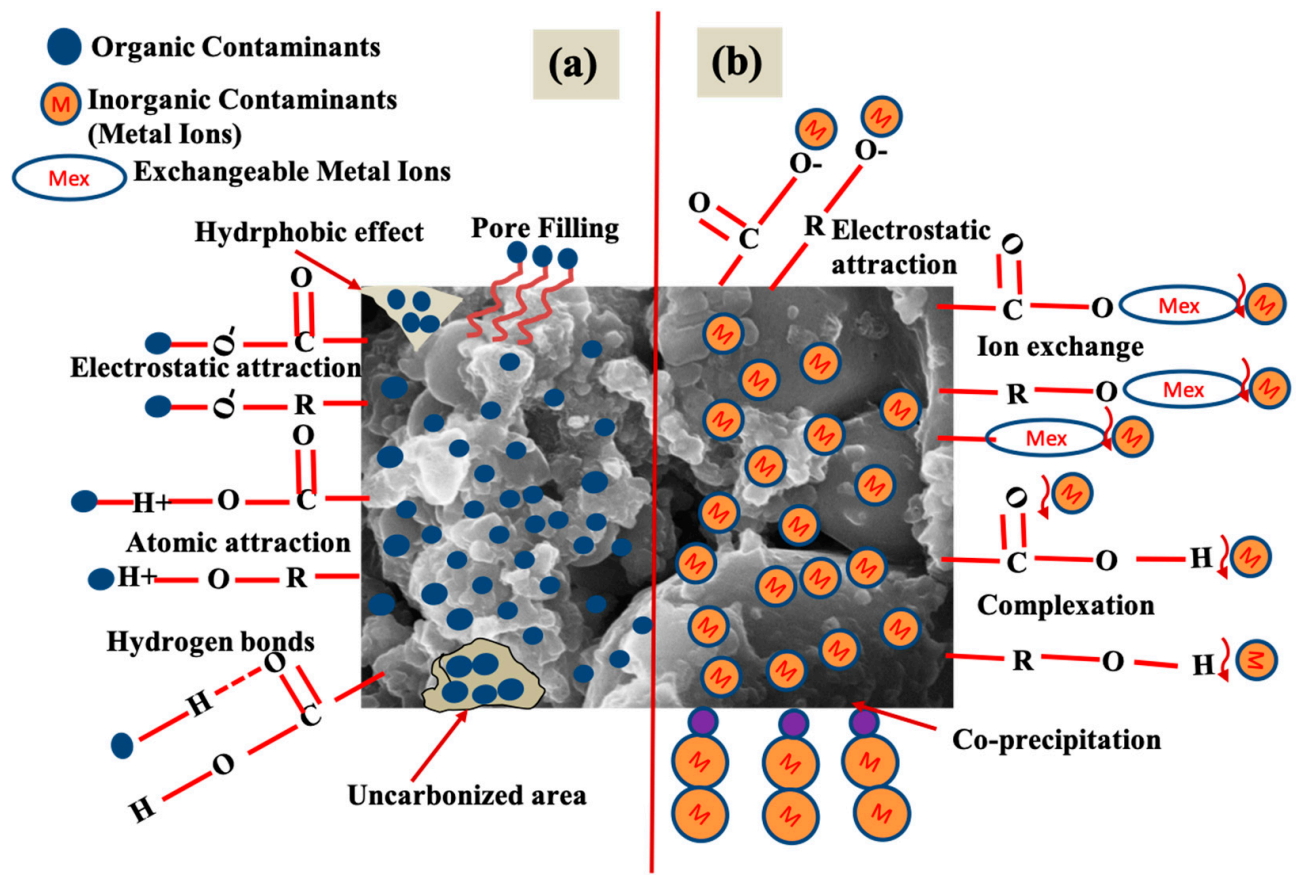

Figure 7. Schematic illustration of the major mechanisms involved in the adsorption of (a) organic pollutants and (b) inorganic pollutants on BCs. 
In general, only physical adsorption, which does not involve the formation of strong chemical bonds, occurs in the adsorption of organic pollutants on BCs. That is, organic molecules attach to the surface of BC through weak bonds, including van der Waals forces, hydrogen bonding, electrostatic forces, and hydrophobic interactions. Van der Waals forces encompass all intermolecular forces that are distance-dependent between the atoms and molecules not associated with covalent or ionic bonds. Adsorption of heavy metals on BCs also occurs through physicochemical interactions, including electrostatic interactions, ion exchange, and complexation, between the metal ions and the functional groups on the $\mathrm{BC}$ surface. Chemical forces resulting from short-range interactions could lead to adsorption of metal ions on the $\mathrm{BC}$ surface through inner-sphere complexation. In addition, due to the change in the chemical conditions in bulk solution or in the micro-environment, precipitation/co-precipitation of heavy metal ions could also occur on the BC surface. Details on the major sorption mechanisms are discussed further below.

\subsubsection{Hydrogen Bonding}

Hydrogen bonding forms through the electrostatic forces between the hydrogen atom (covalently bound to a more electronegative atom or group, e.g., nitrogen, oxygen, and fluorine) and an electronegative atom (as hydrogen bond acceptor) bearing a pair of unshared electrons. As the strength of $\mathrm{H}$-bond is higher than van der Waals forces but less than that of covalent bond, it could play an important role in the sorption of organic pollutants on BCs. BCs manufactured from various feedstocks have abundant oxygen-containing functional groups on their surface, which can act as hydrogen bond donor [31]. Thus, organic pollutants having electronegative elements could attach to the O-containing functional groups of $\mathrm{BC}$ through $\mathrm{H}$-bonding [94]. It has been found that the sorption of organic pollutants (e.g., bisphenol A and 7a-ethinyl estradiol) on the BCs with abundant O-containing surface functional groups was much higher than on those with less O-containing functional groups [2].

\subsubsection{Electrostatic Attraction}

Adsorption of ionizable organic pollutants on the charged surface of BCs occurs through electrostatic attraction. Ionic strength and $\mathrm{pH}$ of the solution have significant effect on the electrostatic interaction between sorbate and sorbent [75]. Reducing solution $\mathrm{pH}$ increases the positive charge of the $\mathrm{BC}$ surface, which could enhance the adsorption of negatively charged organic species. Inyang and co-workers showed that adsorption of methylene blue decreased on BC (from 4.5 to $3 \mathrm{mg} \cdot \mathrm{g}^{-1}$ ) with the increase in solution ion strength (from 0.01 to $0.1 \mathrm{M} \mathrm{NaCl}$ ), which could be attributed to the reduction in electrostatic attraction in the presence of more ions in the solution [97]. Electrostatic interaction was reported to be responsible for the removal of sulfamethazine and norfloxacin by BCs produced from burcucumber (Sicyos angulatus L.), corn stalks, willow branch, and reed stalks $[29,64]$. Overall, electrostatic interaction appears to be important for the adsorption of organic pollutants on plant-based BCs, which might result from the presence of greatersurface charges on them.

\subsubsection{Hydrophobic Interaction}

Hydrophobic interaction originates from the entropic effect brought by destruction of the hydrogen bonds between water molecules by nonpolar solutes in aqueous solution, and drives the assemblage of nonplolar molecules to form anhydrous domains. Hydrophobic interaction is important for the adsorption of neutral or hydrophobic organic pollutants on BCs. Compared to the partitioning mechanism, hydrophobic interaction requires less energy [98]. Adsorption of perfluoro octane sulfonate (PFOS) on BC produced from maize straw was reported to occur through hydrophobic interaction, which was attributed to the highly hydrophobic moiety of the PFOS molecules [99]. In general, BCs produced under higher pyrolysis temperatures have greater adsorption capacities towards non-polar organic pollutants due to the destruction of polar functional groups on the surface of BCs. 


\subsubsection{Anion/Cation Exchange}

The main principle of anion/cation exchange mechanism is the exchange of ionic species on the surface of BCs by the ionizable organic pollutants and cationic metal species [21]. Ion exchange is a nonspecific adsorption process, and involves electrostatic interactions. For heavy metal ions, ion exchange is also considered as outer-sphere complexation, which is weak, but rapid and reversible. The anion/cation exchange capacity of $\mathrm{BC}$ is determined by the functional groups present on its surface, while the adsorption of specific pollutant is also influenced by the valence and radius of the ionic species [100]. Pyrolysis temperature affects the anion/cation exchange capacity of the BC produced, with the anion/cation exchange capacity decreases with the pyrolysis temperature above $350{ }^{\circ} \mathrm{C}$ [98], which probably results from the destruction of O-containing functional groups at high temperatures. It is observed that the presence of more aromatic structure in BCs increases the negative charge in the pi orbital, which creates the opportunity to lose the electrons of existing functional groups, and enhances the adsorption of ionic species through ion exchange [17]. The presence of iron oxides in the feedstock could enhance the ion exchange capacities of BCs. For example, Trakal and co-workers reported that the CECs of $\mathrm{BCs}$ produced from various feedstocks-including grape stalk, nutshell, grape husk, wheat straw, and plum stone-all increased with iron oxide impregnation, which enhanced the adsorption of $\mathrm{Cd}(\mathrm{II})$ and $\mathrm{Pb}(\mathrm{II})$ on them [101].

\subsubsection{Partitioning}

Partitioning of sorbate into the sorbent matrix is driven by the van der Waals forces. $\mathrm{BC}$ can be essentially divided into carbonized (graphene, crystalline) and non-carbonized (amorphous, non-crystalline) fractions, which interact with pollutant molecules differently. Partitioning is the process in which the organic sorbate molecules diffuse into the noncarbonized portion of BC. In general, BCs with higher contents of volatile matter allow greater partitioning of organic pollutants, and thus exhibit higher sorption capacities [102] Partitioning was responsible for the high adsorption capacities of BCs produced from soybean stalk (pyrolysis temperature: $300-700{ }^{\circ} \mathrm{C}$ ), dairy manure (pyrolysis temperature: $200^{\circ} \mathrm{C}$ ), and pig manure (pyrolysis temperature: $350-700^{\circ} \mathrm{C}$ ) towards organic pollutants (phenanthrene and atrazine) $[98,99]$. Partitioning was also the dominant mechanism for adsorption of norflurazon and fluoridone on BCs produced from wood and grass [99].

\subsubsection{Pore Filling}

The micropores $(<2 \mathrm{~nm})$ and the narrow mesopores $(2-50 \mathrm{~nm})$ on the surface of BCs can adsorb organic pollutants through pore filling due to the enhanced dispersion potential within the molecular dimension spaces [98]. The pore filling mechanism depends on the pore size distribution of the $\mathrm{BC}$, with little dependence on the properties of the organic pollutant. Pore filling can be a prominent sorption mechanism when the concentrations of organic pollutants in water are low. It has been reported that adsorption of dyes and carbaryl on BCs produced from rice husk and pig manure occurred primarily through the pore filling mechanism [20,71].

\subsubsection{Complexation}

Complexation is a type of specific adsorption that involves ligand exchange with electron transfer occurring via the bridging ligands. Metal ions can accept the electrons from a range of chelate-forming, electron-donating functional groups present on the surface of $\mathrm{BCs}$, promoting the adsorption of metal ions through complexation [84]. BCs produced at low pyrolysis temperatures have higher tendency to bind heavy metal ions through complexion as they contain more electron-donating functional groups, e.g., carboxyl ($\mathrm{COOH})$, hydroxyl $(-\mathrm{OH})$, and amino $\left(-\mathrm{NH}_{2}\right)[17,21]$. The presence of more oxygen during the pyrolysis of $\mathrm{BC}$ can increase the adsorption of metal ions through complexion due to the greater extent of surface oxidation. It is observed that BCs produced from plant feedstocks have higher complexation affinity towards heavy metals (e.g., $\mathrm{Cr}, \mathrm{Pb}, \mathrm{Zn}$, 
$\mathrm{Ni}, \mathrm{Hg}, \mathrm{Cu}$, and $\mathrm{As}$ ) than those produced from animal sources, e.g., poultry litter and dairy manure $[5,83,84]$. This is attributed to the greater abundance of oxygenic functional groups present in plant-based BCs compared to the latter. Sophisticated spectroscopic techniques, such as Fourier transform infrared spectroscopy (FTIR), X-ray diffraction (XRD), scanning electron microscope (SEM), energy-dispersive X-ray (EDX), X-ray photoelectron spectroscopy (XPS), and transmission electron microscopy (TEM) can help reveal more insights on the binding mechanism of heavy metal ions on BCs and better understand the contribution of complexation to the overall sorption.

\subsubsection{Precipitation/Co-Precipitation}

Precipitation/co-precipitation is an important mechanism responsible for the adsorption of heavy metals onto BCs [21]. Dissolution of the mineral components in BCs alters the chemistry of water and soil solution, which may cause precipitation/co-precipitation of the heavy metals present. It has been reported that the $\mathrm{pH}$ of soil could increase after application of BCs, which are alkaline, and precipitation of heavy metal ions in the forms of hydroxide, phosphate, and carbonate salts could occur [103]. The type of feedstock and pyrolysis temperature affect the alkalinity of the $\mathrm{BC}$ produced, and thus the contribution of precipitation in the adsorption of heavy metals. BCs produced from cellulose and hemicellulose materials under relatively high pyrolysis temperatures $\left(>300^{\circ} \mathrm{C}\right)$ have high alkalinity and tend to induce mineral precipitation, either on the surface of the BCs or in the solution [104]. BCs produced from sugar beet tailing, banana peels, dairy manure, and residue of biogas production were shown to be effective in the removal of heavy metals $(\mathrm{Cr}, \mathrm{Pb}, \mathrm{Zn}$, and $\mathrm{Ni})$ through inducing their precipitation [83,85].

\section{Conclusions and Future Prospects}

With high specific surface areas, aromatic surface, and multiple functional groups, BCs can efficiently remove a range of organic and inorganic pollutants, such as pesticides, synthetic dyes, and heavy metals from water and soils. Although BCs are less efficient than activated carbon, they could be produced from a wide variety of organic waste at low cost, and the use of $\mathrm{BCs}$ in pollution control has received growing interests. The types of feedstock and the pyrolysis temperature significantly impact the physical and chemical properties of the BC produced, and thus its affinity towards organic and inorganic pollutants. Hydrogen bonding, pore filling, electrostatic attraction, hydrophobic interaction, and van der Waals forces can be involved in the adsorption of organic pollutants from water and soils by BC, whereas ion exchange, precipitation, electrostatic attraction, and complexation are among the major mechanisms responsible for the removal of inorganic pollutants by them. Despite the significant advances that have been made in the development of BCs/E-BCs and understanding of the mechanisms of pollutant adsorption on them, more research and development efforts are still needed to make the application of $\mathrm{BCs} / \mathrm{E}-\mathrm{BC}$ in environmental remediation cost-effective. Attention should be paid to the following research priorities to make $\mathrm{BCs} / \mathrm{E}-\mathrm{BCs}$ viable for environmental remediation:

- The relationships between the physical and chemical properties of BCs and their feedstock type and pyrolysis temperature are still not fully understood. More study is needed to characterize the morphology and structure of BCs, their chemical compositions and surface functional groups to link them with their production conditions (i.e., feedstock and pyrolysis temperature). Such understanding will help to guide the production of BCs with suitable properties for the removal of the targeted pollutants.

- Many studies have reported promising results on the use of BCs/E-BCs in removing organic and inorganic pollutants from water and soils. However, most of these studies were conducted in the lab or as small pilot tests under well controlled conditions. Large scale pilot tests and field studies on the applications of BCs/E-BCs in pollution control are necessary to evaluate their performance. In particular, the long-term effectiveness of $\mathrm{BCs} / \mathrm{E}-\mathrm{BC}$ in retaining pollutants, and the effect of natural aging and 
precipitation on the stability of the BCs/E-BCs and the adsorbed pollutants should be quantified.

- Similar to activated carbon, once the adsorption capacities of the BCs/E-BCs are saturated, they can no longer be effectively used. In the treatment of water, the "spent" BCs/E-BCs need to be separated from the aqueous phase, which can be subsequently regenerated and reused. Pyrolysis treatment may cause degradation of the sorbed organic pollutants on BCs/E-BCs and thus efficiently regenerate their adsorption capacities. In contrast, desorption of the heavy metals from BCs/E-BCs would unavoidably generate a stream of secondary waste that needs to be properly disposed of. For BC/E-BC amended soils, separation of the BC/E-BC particles from the soil mixtures is not practical, and their physical and chemical stability is critical for the immobilization of the pollutants sorbed on them. Thus, the stability, regeneration, and reuse of $\mathrm{BCs} / \mathrm{E}-\mathrm{BCs}$ should be further improved to enhance their performance in environmental remediation.

- For any environmental remediation technologies, cost is always a very important factor. Production of BCs and their modification through various methods should be assessed in terms of cost, and the use of alternative waste organic materials as $\mathrm{BC}$ feedstocks should be explored to lower their production cost. With surface modification and functionalization, E-BCs can have enhanced adsorption capacities for the targeted pollutants, while BCs can also be modified to increase their recyclability and facilitate the separation of the adsorbed pollutants. More research is needed to develop eco-friendly synthesis processes of E-BC nanocomposites using green waste materials and evaluate the performance of such nanocomposites as sorbents in environmental remediation.

- A limited number of studies have shown that some BCs have an adverse effect on the microorganisms, plants, and fauna in soils. Thus, thorough examination of environmental risk of the BCs produced from various feedstocks and under different pyrolysis temperatures, as well as the E-BCs modified with different methods, should be conducted before applying them in environmental remediation.

Author Contributions: Conceptualization, T.I. and H.C.; Writing-original draft preparation, T.I., Y.L. and H.C.; Writing-review and editing, T.I., Y.L. and H.C. All authors have read and agreed to the published version of the manuscript.

Funding: This work was funded by the Natural Science Foundation of China (grant nos. U2006212 and 41725015).

Institutional Review Board Statement: Not applicable.

Informed Consent Statement: Not applicable.

Conflicts of Interest: The authors declare no conflict of interest.

\section{References}

1. Wang, H.; Guo, W.; Yin, R.; Du, J.; Wu, Q.; Luo, H.; Liu, B.; Seguya, F.; Ren, N. Biochar-induced Fe(III) reduction for persulfate activation in sulfamethoxazole degradation: Insight into the electron transfer, radical oxidation and degradation pathways. Chem. Eng. J. 2019, 362, 561-569. [CrossRef]

2. Zheng, H.; Zhang, C.; Liu, B.; Liu, G.; Zhao, M.; Xu, G.; Luo, X.; Li, F.; Xing, B. Biochar for water and soil remediation: Production, characterization, and application. In A New Paradigm for Environmental Chemistry and Toxicology; Jiang, G., Li, X., Eds.; Springer: Singapore, 2019; pp. 153-196.

3. Vilela, C.L.S.; Bassin, J.P.; Peixoto, R.S. Water contamination by endocrine disruptors: Impacts, microbiological aspects and trends for environmental protection. Environ. Pollut. 2018, 235, 546-559. [CrossRef] [PubMed]

4. Yang, X.; Wan, Y.; Zheng, Y.; He, F.; Yu, Z.; Huang, J.; Wang, H.; Ok, Y.S.; Jiang, Y.; Gao, B. Surface functional groups of carbon-based adsorbents and their roles in the removal of heavy metals from aqueous solutions: A critical review. Chem. Eng. J. 2019, 366, 608-621. [CrossRef]

5. Ahmad, M.; Rajapaksha, A.U.; Lim, J.E.; Zhang, M.; Bolan, N.; Mohan, D.; Vithanage, M.; Lee, S.S.; Ok, Y.S. Biochar as a sorbent for contaminant management in soil and water: A review. Chemosphere 2014, 99, 19-33. [CrossRef] 
6. Ippolito, J.A.; Cui, L.; Kammann, C.; Wrage-Monnig, N.; Estavillo, J.M.; Mendizabal, T.F.; Cayuela, M.L.; Sigua, G.; Novak, J.; Spokas, K.; et al. Feedstock choice, pyrolysis temperature and type influence biochar characteristics: A comprehensive meta-data analysis review. Biochar 2020, 2, 421-438. [CrossRef]

7. Smith, P.; Adams, J.; Beerling, D.J.; Beringer, T.; Calvin, K.V.; Fuss, S.; Griscom, B.; Hagemann, N.; Kammann, C.; Kraxner, F.; et al. Impacts of land-based greenhouse gas removal options on ecosystem services and the United Nations Sustainable Development Goals. Annu. Rev. Environ. Resour. 2019, 44, 255-286. [CrossRef]

8. Yuan, P.; Wang, J.; Pan, Y.; Shen, B.; Wu, C. Review of biochar for the management of contaminated soil: Preparation, application and prospect. Sci. Total Environ. 2019, 659, 473-490. [CrossRef]

9. Park, J.H.; Ok, Y.S.; Kim, S.H.; Cho, J.S.; Heo, J.S.; Delaune, R.D.; Seo, D.C. Competitive adsorption of heavy metals onto sesame straw biochar in aqueous solutions. Chemosphere 2016, 142, 77-83. [CrossRef]

10. Novak, J.M.; Sigua, G.C.; Ducey, T.F.; Watts, D.W.; Stone, K.C. Designer biochars impact on corn grain yields, biomass production, and fertility properties of a highly-weathered Ultisol. Environments 2019, 6, 64. [CrossRef]

11. Ippolito, J.A.; Spokas, K.A.; Novak, J.M.; Lentz, R.D.; Cantrell, K.B. Biochar elemental composition and factors influencing nutrient retention. In Biochar for Environ mental Management: Science, Technology and Implementation, 2nd ed.; Lehmann, J., Joseph, S., Eds.; Routledge: New York, NY, USA, 2015; pp. 137-161.

12. Nguyen, T.T.N.; Xu, C.-Y.; Tahmasbian, I.; Che, R.; Xu, Z.; Zhou, X.; Wallace, H.; Bai, S.H. Effects of biochar on soil available inorganic nitrogen: A review and meta-analysis. Geoderma 2017, 288, 79-96. [CrossRef]

13. Noreen, S.; Abd-Esalam, K.A. Biochar-based nanocomposites: A sustainable tool in wastewater bioremediation. In Aquananotechnology: Applications of Nanomaterials for Water Purification; Abd-Elsalam, K.A., Zahid, M., Eds.; Elsevier: Amsterdam, The Netherlands, 2021; pp. 185-200.

14. Thompson, K.A.; Shimabuku, K.K.; Kearns, J.P.; Knappe, D.R.U.; Summers, R.S.; Cook, S.M. Environmental comparison of biochar and activated carbon for tertiary wastewater treatment. Environ. Sci. Technol. 2016, 50, 11253-11262. [CrossRef]

15. Kambo, H.S.; Dutta, A. A comparative review of biochar and hydrochar in terms of production, physico-chemical properties and applications. Renew. Sustain. Energy Rev. 2015, 45, 359-378. [CrossRef]

16. Tan, X.; Liu, Y.-G.; Gu, Y.-L.; Xu, Y.; Zeng, G.-M.; Hu, X.-J.; Liu, S.-B.; Wang, X.; Liu, S.-M.; Li, J. Biochar-based nano-composites for the decontamination of wastewater: A review. Bioresour. Technol. 2016, 212, 318-333. [CrossRef]

17. Li, R.; Wang, J.; Zhou, B.; Zhang, Z.; Liu, S.; Lei, S.; Xiao, R. Simultaneous capture removal of phosphate, ammonium and organic substances by $\mathrm{MgO}$ impregnated biochar and its potential use in swine wastewater treatment. J. Clean. Prod. 2017, 147, 96-107. [CrossRef]

18. Chaukura, N.; Murimba, E.C.; Gwenzi, W. Synthesis, characterisation and methyl orange adsorption capacity of ferric oxidebiochar nanocomposites derived from pulp and paper sludge. Appl. Water Sci. 2017, 7, 2175-2186. [CrossRef]

19. Bhushan, B.; Gupta, V.; Kotnala, S. Development of magnetic-biochar nano-composite: Assessment of its physico-chemical properties. Mater. Today Proc. 2020, 26, 3271-3274. [CrossRef]

20. Islam, T.; Peng, C.; Ali, I.; Li, J.; Khan, Z.M.; Sultan, M.; Naz, I. Synthesis of rice husk derived magnetic bio-char through liquefaction to adsorb anionic and cationic dyes from aqueous solutions. Arab. J. Sci. Eng. 2021, 46, 233-246. [CrossRef]

21. Wang, Y.; Zhong, B.; Shafi, M.; Ma, J.; Guo, J.; Wu, J.; Ye, Z.; Liu, D.; Jin, H. Effects of biochar on growth, and heavy metals accumulation of moso bamboo (Phyllostachy pubescens), soil physical properties, and heavy metals solubility in soil. Chemosphere 2019, 219, 510-516. [CrossRef]

22. Li, S.; Chan, C.Y.; Sharbatmaleki, M.; Trejo, H.; Delagah, S. Engineered biochar production and its potential benefits in a closed-loop water-reuse agricultural system. Water 2020, 12, 2847. [CrossRef]

23. Li, J.; Dai, J.J.; Liu, G.Q.; Zhang, H.D.; Gao, Z.P.; Fu, J.; He, Y.F.; Huang, Y. Biochar from microwave pyrolysis of bio-mass: A review. Biomass Bioenergy 2016, 94, 228-244. [CrossRef]

24. Lee, J.; Cho, W.-C.; Poo, K.-M.; Choi, S.; Kim, T.-N.; Son, E.-B.; Choi, Y.-J.; Kim, Y.M.; Chae, K.-J. Refractory oil wastewater treatment by dissolved air flotation, electrochemical advanced oxidation process, and magnetic biochar integrated system. $J$. Water Process. Eng. 2020, 36, 101358. [CrossRef]

25. Lyu, H.H.; Gao, B.; He, F.; Zimmerman, A.R.; Ding, C.; Huang, H.; Tang, J.C. Effects of ball milling on the physico-chemical and sorptive properties of biochar: Experimental observations and governing mechanisms. Environ. Pollut. 2018, $233,54-63$. [CrossRef]

26. Yi, Y.Q.; Huang, Z.X.; Lu, B.Z.; Xian, J.Y.; Tsang, E.P.; Cheng, W.; Fang, J.Z.; Fang, Z.Q. Magnetic biochar for environmental remediation: A review. Bioresour. Technol. 2020, 298, 122468. [CrossRef]

27. Sajjadi, B.; Zubatiuk, T.; Leszczynska, D.; Leszczynski, J.; Chen, W.Y. Chemical activation of biochar for energy and en-vironmental applications: A comprehensive review. Rev. Chem. Eng. 2019, 35, 777-815. [CrossRef]

28. Gupta, R.K.; Dubey, M.; Kharel, P.; Gu, Z.; Fan, Q.H. Biochar activated by oxygen plasma for supercapacitors. J. Power Sources 2015, 274, 1300-1305. [CrossRef]

29. Wang, B.; Jiang, Y.-S.; Li, F.-Y.; Yang, D.-Y. Preparation of biochar by simultaneous carbonization, magnetization and activation for norfloxacin removal in water. Bioresour. Technol. 2017, 233, 159-165. [CrossRef]

30. Wang, B.; Gao, B.; Fang, J. Recent advances in engineered biochar productions and applications. Crit. Rev. Environ. Sci. Technol. 2017, 47, 2158-2207. [CrossRef] 
31. Dai, Y.; Zhang, N.; Xing, C.; Cui, Q.; Sun, Q. The adsorption, regeneration and engineering applications of biochar for removal organic pollutants: A review. Chemosphere 2019, 223, 12-27. [CrossRef] [PubMed]

32. Zhang, Y.; Cao, B.; Zhao, L.; Sun, L.; Gao, Y.; Li, J.; Yang, F. Biochar-supported reduced graphene oxide composite for adsorption and coadsorption of atrazine and lead ions. Appl. Surf. Sci. 2018, 427, 147-155. [CrossRef]

33. Yang, X.; Zhang, S.; Ju, M.; Liu, L. Preparation and modification of biochar materials and their application in soil remediation. Appl. Sci. 2019, 9, 1365. [CrossRef]

34. Tomczyk, A.; Sokołowska, Z.; Boguta, P. Biochar physicochemical properties: Pyrolysis temperature and feedstock kind effects. Rev. Environ. Sci. BioTechnol. 2020, 19, 191-215. [CrossRef]

35. Keiluweit, M.; Nico, P.; Johnson, M.G.; Kleber, M. Dynamic molecular structure of plant biomass-derived black carbon (biochar). Environ. Sci. Technol. 2010, 44, 1247-1253. [CrossRef]

36. Chen, B.; Chen, Z. Sorption of naphthalene and 1-naphthol by biochars of orange peels with different pyrolytic temperatures. Chemosphere 2009, 76, 127-133. [CrossRef]

37. Uchimiya, M.; Chang, S.; Klasson, K.T. Screening biochars for heavy metal retention in soil: Role of oxygen functional groups. J. Hazard. Mater. 2011, 190, 432-441. [CrossRef]

38. Ahmad, M.; Lee, S.S.; Dou, X.; Mohan, D.; Sung, J.-K.; Yang, J.E.; Ok, Y.S. Effects of pyrolysis temperature on soybean stover- and peanut shell-derived biochar properties and TCE adsorption in water. Bioresour. Technol. 2012, 118, 536-544. [CrossRef]

39. Kloss, S.; Zehetner, F.; Dellantonio, A.; Hamid, R.; Ottner, F.; Liedtke, V.; Schwanninger, M.; Gerzabek, M.; Soja, G. Characterization of slow pyrolysis biochars: Effects of feedstocks and pyrolysis temperature on biochar properties. J. Environ. Qual. 2012, 41, 990-1000. [CrossRef]

40. Karaosmanoğlu, F.; Işıḡıgür-Ergüdenler, A.; Sever, A. Biochar from the straw-stalk of rapeseed plant. Energy Fuels 2000, 14, 336-339. [CrossRef]

41. Tong, X.-J.; Li, J.-Y.; Yuan, J.; Xu, R.-K. Adsorption of $\mathrm{Cu}(\mathrm{II})$ by biochars generated from three crop straws. Chem. Eng. J. 2011, 172, 828-834. [CrossRef]

42. Liu, P.; Liu, W.-J.; Jiang, H.; Chen, J.-J.; Li, W.-W.; Yu, H.-Q. Modification of bio-char derived from fast pyrolysis of biomass and its application in removal of tetracycline from aqueous solution. Bioresour. Technol. 2012, 121, 235-240. [CrossRef]

43. Lee, J.W.; Kidder, M.; Evans, B.R.; Paik, S.; Buchanan, A.C., III; Garten, C.T.; Brown, R.C. Characterization of biochars produced from corn stovers for soil amendment. Environ. Sci. Technol. 2010, 44, 7970-7974. [CrossRef]

44. Mullen, C.A.; Boateng, A.A.; Goldberg, N.M.; Lima, I.M.; Laird, D.A.; Hicks, K.B. Bio-oil and bio-char production from corn cobs and stover by fast pyrolysis. Biomass Bioenergy 2010, 34, 67-74. [CrossRef]

45. Liu, Z.; Zhang, F.-S.; Wu, J. Characterization and application of chars produced from pinewood pyrolysis and hydrothermal treatment. Fuel 2010, 89, 510-514. [CrossRef]

46. Lian, F.; Huang, F.; Chen, W.; Xing, B.; Zhu, L. Sorption of apolar and polar organic contaminants by waste tire rubber and its chars in single- and bi-solute systems. Environ. Pollut. 2011, 159, 850-857. [CrossRef]

47. Cantrell, K.B.; Hunt, P.G.; Uchimiya, M.; Novak, J.M.; Ro, K. Impact of pyrolysis temperature and manure source on physicochemical characteristics of biochar. Bioresour. Technol. 2012, 107, 419-428. [CrossRef] [PubMed]

48. Ro, K.S.; Cantrell, K.B.; Hunt, P.G. High-temperature pyrolysis of blended animal manures for producing renewable energy and value-added biochar. Ind. Eng. Chem. Res. 2010, 49, 10125-10131. [CrossRef]

49. Eibisch, N.; Durner, W.; Bechtold, M.; Fuß, R.; Mikutta, R.; Woche, S.K.; Helfrich, M. Does water repellency of pyrochars and hydrochars counter their positive effects on soil hydraulic properties? Geoderma 2015, 245-246, 31-39. [CrossRef]

50. Kinney, T.J.; Masiello, C.A.; Dugan, B.; Hockaday, W.C.; Dean, M.R.; Zygourakis, K.; Barnes, R.T. Hydrologic properties of biochars produced at different temperatures. Biomass Bioenergy 2012, 41, 34-43. [CrossRef]

51. Krasucka, P.; Pan, B.; Ok, Y.S.; Mohan, D.; Sarkar, B.; Oleszczuk, P. Engineered biochar-A sustainable solution for the removal of antibiotics from water. Chem. Eng. J. 2021, 405, 126926. [CrossRef]

52. Waqas, M.; Aburiazaiza, A.; Miandad, R.; Rehan, M.; Barakat, M.; Nizami, A. Development of biochar as fuel and catalyst in energy recovery technologies. J. Clean. Prod. 2018, 188, 477-488. [CrossRef]

53. Oni, B.A.; Oziegbe, O.; Olawole, O.O. Significance of biochar application to the environment and economy. Ann. Agric. Sci. 2019, 64, 222-236. [CrossRef]

54. Ali, N.; Khan, S.; Li, Y.; Zheng, N.; Yao, H. Influence of biochars on the accessibility of organochlorine pesticides and microbial com- munity in contaminated soils. Sci. Total Environ. 2019, 647, 551-560. [CrossRef]

55. Huang, S.; Bao, J.; Shan, M.; Qin, H.; Wang, H.; Yu, X.; Chen, J.; Xu, Q. Dynamic changes of polychlorinated biphenyls (PCBs) degradation and adsorption to biochar as affected by soil organic carbon content. Chemosphere 2018, 211, 120-127. [CrossRef] [PubMed]

56. He, L.; Fan, S.; Mueller, K.; Hu, G.; Huang, H.; Zhang, X.; Lin, X.; Che, L.; Wang, H. Biochar reduces the bioavailability of di-(2-ethylhexyl) phthalate in soil. Chemosphere 2016, 142, 24-27. [CrossRef] [PubMed]

57. Williams, M.; Martin, S.; Kookana, R. Sorption and plant uptake of pharmaceuticals from an artificially contaminated soil amended with biochars. Plant Soil 2015, 395, 75-86. [CrossRef]

58. Saletnik, B.; Zaguła, G.; Bajcar, M.; Tarapatskyy, M.; Bobula, G.; Puchalski, C. Biochar as a multifunctional component of the environment-A review. Appl. Sci. 2019, 9, 1139. [CrossRef] 
59. Cho, D.-W.; Yoon, K.; Ahn, Y.; Sun, Y.; Tsang, D.C.W.; Hou, D.; Ok, Y.S.; Song, H. Fabrication and environmental applications of multifunctional mixed metal-biochar composites (MMBC) from red mud and lignin wastes. J. Hazard. Mater. 2019, 374, 412-419. [CrossRef]

60. Chen, B.; Zhou, D.; Zhu, L. Transitional adsorption and partition on nonpolar and polar aromatic contaminants by bio-chars of pine needles with different pyrolytic temperatures. Environ. Sci. Technol. 2008, 42, 5137-5143. [CrossRef]

61. Sun, K.; Ro, K.; Guo, M.; Novak, J.; Mashayekhi, H.; Xing, B. Sorption of bisphenol A, $17 \alpha$-ethinyl estradiol and phenanthrene on thermally and hydrothermally produced biochars. Bioresour. Technol. 2011, 102, 5757-5763. [CrossRef]

62. Kong, H.; He, J.; Gao, Y.; Wu, H.; Zhu, X. Cosorption of phenanthrene and mercury(II) from aqueous solution by soy-bean stalk-based biochar. J. Agric. Food Chem. 2011, 59, 12116-12123. [CrossRef]

63. Rajapaksha, A.U.; Vithanage, M.; Zhang, M.; Ahmad, M.; Mohan, D.; Chang, S.X.; Ok, Y.S. Pyrolysis condition affected sulfamethazine sorption by tea waste biochars. Bioresour. Technol. 2014, 166, 303-308. [CrossRef]

64. Rajapaksha, A.U.; Vithanage, M.; Ahmad, M.; Seo, D.-C.; Cho, J.-S.; Lee, S.-E.; Lee, S.S.; Ok, Y.S. Enhanced sulfamethazine removal by steam-activated invasive plant-derived biochar. J. Hazard. Mater. 2015, 290, 43-50. [CrossRef] [PubMed]

65. Xu, R.-K.; Xiao, S.-C.; Yuan, J.; Zhao, A.-Z. Adsorption of methyl violet from aqueous solutions by the biochars derived from crop residues. Bioresour. Technol. 2011, 102, 10293-10298. [CrossRef] [PubMed]

66. Yang, X.-B.; Ying, G.-G.; Peng, P.-A.; Wang, L.; Zhao, J.-L.; Zhang, L.-J.; Yuan, P.; He, H.-P. Influence of biochars on plant uptake and dissipation of two pesticides in an agricultural soil. J. Agric. Food Chem. 2010, 58, 7915-7921. [CrossRef]

67. Yao, Y.; Gao, B.; Chen, H.; Jiang, L.; Inyang, M.; Zimmerman, A.R.; Cao, X.; Yang, L.; Xue, Y.; Li, H. Adsorption of sulfamethoxazole on biochar and its impact on reclaimed water irrigation. J. Hazard. Mater. 2012, 209-210, 408-413. [CrossRef]

68. Yu, X.; Pan, L.; Ying, G.; Kookana, R.S. Enhanced and irreversible sorption of pesticide pyrimethanil by soil amended with biochars. J. Environ. Sci. 2010, 22, 615-620. [CrossRef]

69. Hale, S.; Hanley, K.; Lehmann, J.; Zimmerman, A.; Cornelissen, G. Effects of chemical, biological, and physical aging as well as soil addition on the sorption of pyrene to activated carbon and biochar. Environ. Sci. Technol. 2011, 45, 10445-10453. [CrossRef]

70. Cao, X.; Harris, W. Properties of dairy-manure-derived biochar pertinent to its potential use in remediation. Bioresour. Technol. 2010, 101, 5222-5228. [CrossRef]

71. Zhang, P.; Sun, H.; Yu, L.; Sun, T. Adsorption and catalytic hydrolysis of carbaryl and atrazine on pig manure-derived biochars: Impact of structural properties of biochars. J. Hazard. Mater. 2013, 244-245, 217-224. [CrossRef] [PubMed]

72. Uchimiya, M.; Lima, I.M.; Klasson, K.T.; Chang, S.; Wartelle, L.H.; Rodgers, J.E. Immobilization of heavy metal ions (Cu ${ }^{\text {II }}$, Cd ${ }^{\text {II }}$, $\mathrm{Ni}^{\mathrm{II}}$, and $\mathrm{Pb}^{\mathrm{II}}$ ) by broiler litter-derived biochars in water and soil. J. Agric. Food Chem. 2010, 58, 5538-5544. [CrossRef]

73. Guo, M. The 3R principles for applying biochar to improve soil health. Soil Syst. 2020, 4, 9. [CrossRef]

74. Jones, D.L.; Jones, G.E.; Murphy, D.V. Biochar mediated alternations in herbicide breakdown and leaching in soil. Soil Biol. Biochem. 2011, 43, 804-813. [CrossRef]

75. Guo, M.; Song, W.; Tian, J. Biochar-facilitated soil remediation: Mechanisms and efficacy variations. Front. Environ. Sci. 2020, 8 , 521512. [CrossRef]

76. Irfan, M.; Hussain, Q.; Khan, K.S.; Akmal, M.; Ijaz, S.S.; Hayat, R. Response of soil microbial biomass and enzymatic activity to biochar amendment in the organic carbon deficient arid soil: A 2-year field study. Arab. J. Geosci. 2019, 12, 95. [CrossRef]

77. Xu, T.; Lou, L.; Luo, L.; Cao, R.; Duan, D.; Chen, Y. Effect of bamboo biochar on pentachlorophenol leachability and bioavailability in agricultural soil. Sci. Total Environ. 2012, 414, 727-731. [CrossRef]

78. Zhang, H.; Lin, K.; Wang, H.; Gan, J. Effect of Pinus radiata derived biochars on soil sorption and desorption of phenanthrene. Environ. Pollut. 2010, 158, 2821-2825. [CrossRef] [PubMed]

79. Denyes, M.J.; Langlois, V.S.; Rutter, A.; Zeeb, B.A. The use of biochar to reduce soil PCB bioavailability to Cucurbita pepo and Eisenia fetida. Sci. Total Environ. 2012, 437, 76-82. [CrossRef]

80. Beesley, L.; Jiménez, E.M.; Eyles, J.L.G. Effects of biochar and greenwaste compost amendments on mobility, bioavailability and toxicity of inorganic and organic contaminants in a multi-element polluted soil. Environ. Pollut. 2010, 158, 2282-2287. [CrossRef] [PubMed]

81. Cao, X.; Ma, L.; Liang, Y.; Gao, B.; Harris, W. Simultaneous immobilization of lead and atrazine in contaminated soils using dairy-manure biochar. Environ. Sci. Technol. 2011, 45, 4884-4889. [CrossRef]

82. Martin, S.M.; Kookana, R.S.; Van Zwieten, L.; Krull, E. Marked changes in herbicide sorption-desorption upon ageing of biochars in soil. J. Hazard. Mater. 2012, 231-232, 70-78. [CrossRef]

83. Ahmad, Z.; Gao, B.; Mosa, A.; Yu, H.; Yin, X.; Bashir, A.; Ghoveisi, H.; Wang, S. Removal of Cu(II), Cd(II) and Pb(II) ions from aqueous solutions by biochars derived from potassium-rich biomass. J. Clean. Prod. 2018, 180, 437-449. [CrossRef]

84. Ifthikar, J.; Jiao, X.; Ngambia, A.; Wang, T.; Khan, A.; Jawad, A.; Xue, Q.; Liu, L.; Chen, Z. Facile one-pot synthesis of sustainable carboxymethyl chitosan-Sewage sludge biochar for effective heavy metal chelation and regeneration. Bioresour. Technol. 2018, 262, 22-31. [CrossRef]

85. Bogusz, A.; Nowak, K.; Stefaniuk, M.; Dobrowolski, R.; Oleszczuk, P. Synthesis of biochar from residues after biogas production with respect to cadmium and nickel removal from wastewater. J. Environ. Manag. 2017, 201, 268-276. [CrossRef] [PubMed]

86. Kumar, A.; Kumar, A.; Sharma, G.; Naushad, M.; Stadler, F.J.; Ghfar, A.A.; Dhiman, P.; Saini, R.V. Sustainable nanohybrids of magnetic biochar supported g-C3N4/FeVO4 for solar-powered degradation of noxious pollutants-Synergism of adsorption, photocatalysis and photoozonation. J. Clean. Prod. 2017, 165, 431-451. [CrossRef] 
87. Liu, Z.; Zhang, F.-S. Removal of lead from water using biochars prepared from hydrothermal liquefaction of biomass. J. Hazard. Mater. 2009, 167, 933-939. [CrossRef] [PubMed]

88. Mohan, D.; Rajput, S.; Singh, V.K.; Steele, P.H.; Pittman, C.U., Jr. Modeling and evaluation of chromium remediation from water using low cost biochar, a green adsorbent. J. Hazard. Mater. 2011, 188, 319-333. [CrossRef]

89. Xu, X.; Cao, X.; Zhao, L.; Wang, H.; Yu, H.; Gao, B. Removal of $\mathrm{Cu}, \mathrm{Zn}$, and $\mathrm{Cd}$ from aqueous solutions by the dairy manure-derived biochar. Environ. Sci. Pollut. Res. 2013, 20, 358-368. [CrossRef]

90. Wu, C.; Shi, L.; Xue, S.; Li, W.; Jiang, X.; Rajendran, M.; Qian, Z. Effect of sulfuriron modified biochar on the available cadmium and bacterial community structure in contaminated soils. Sci. Total Environ. 2019, 647, 1158-1168. [CrossRef] [PubMed]

91. Boostani, H.R.; Najafi-Ghiri, M.; Mirsoleimani, A. The effect of biochars application on reducing the toxic effects of nickel and growth indices of spinach (Spinacia oleracea L.) in a calcareous soil. Environ. Sci. Pollut. Res. 2018, 26, 1751-1760. [CrossRef] [PubMed]

92. Nie, C.; Yang, X.; Niazi, N.K.; Xu, X.; Wen, Y.; Rinklebe, J.; Ok, Y.S.; Xu, S.; Wang, H. Impact of sugarcane bagasse-derived biochar on heavy metal availability and microbial activity: A field study. Chemosphere 2018, 200, 274-282. [CrossRef]

93. Shen, Z.; Som, A.M.; Wang, F.; Jin, F.; McMillan, O.; Al-Tabbaa, A. Long-term impact of biochar on the immobilisation of nickel (II) and zinc (II) and the revegetation of a contaminated site. Sci. Total Environ. 2016, 542, 771-776. [CrossRef] [PubMed]

94. Namgay, T.; Singh, B.; Singh, B.P. Influence of biochar application to soil on the availability of As, Cd, Cu, Pb, and Zn to maize (Zea mays L.). Soil. Res. 2010, 48, 638-647. [CrossRef]

95. Jiang, T.-Y.; Xu, R.-K.; Gu, T.-X.; Jiang, J. Effect of crop-straw derived biochars on $\mathrm{Pb}(\mathrm{II})$ adsorption in two variable charge soils. J. Integr. Agric. 2014, 13, 507-516. [CrossRef]

96. Hartley, W.; Dickinson, N.M.; Riby, P.; Lepp, N.W. Arsenic mobility in brownfield soils amended with green waste compost or biochar and planted with Miscanthus. Environ. Pollut. 2009, 157, 2654-2662. [CrossRef] [PubMed]

97. Inyang, M.; Gao, B.; Zimmerman, A.; Zhang, M.; Chen, H. Synthesis, characterization, and dye sorption ability of carbon nanotube-biochar nanocomposites. Chem. Eng. J. 2014, 236, 39-46. [CrossRef]

98. Ambaye, T.G.; Vaccari, M.; Van Hullebusch, E.D.; Amrane, A.; Rtimi, S. Mechanisms and adsorption capacities of biochar for the removal of organic and inorganic pollutants from industrial wastewater. Int. J. Environ. Sci. Technol. 2020, 1-22. [CrossRef]

99. Chen, B.; Chen, Z.; Lv, S. A novel magnetic biochar efficiently sorbs organic pollutants and phosphate. Bioresour. Technol. 2011, 102, 716-723. [CrossRef] [PubMed]

100. Rizwan, M.; Ali, S.; Qayyum, M.F.; Ibrahim, M.; Rehman, M.Z.; Abbas, T.; Ok, Y.S. Mechanisms of biochar-mediated alleviation of toxicity of trace elements in plants: A critical review. Environ. Sci. Pollut. Res. 2016, 23, 2230-2248. [CrossRef] [PubMed]

101. Trakal, L.; Veselská, V.; Šafařík, I.; Vítková, M.; Číhalová, S.; Komárek, M. Lead and cadmium sorption mechanisms on magnetically modified biochars. Bioresour. Technol. 2016, 203, 318-324. [CrossRef]

102. Liu, G.; Zheng, H.; Jiang, Z.; Zhao, J.; Wang, Z.; Pan, B.; Xing, B. Formation and physicochemical characteristics of nano biochar: Insight into chemical and colloidal stability. Environ. Sci. Technol. 2018, 52, 10369-10379. [CrossRef]

103. Fidel, R.B.; Laird, D.A.; Spokas, K.A. Sorption of ammonium and nitrate to biochars is electrostatic and pH-dependent. Sci. Rep. 2018, 8, 17627. [CrossRef]

104. Banik, C.; Lawrinenko, M.; Bakshi, S.; Laird, D.A. Impact of pyrolysis temperature and feedstock on surface charge and functional group chemistry of biochars. J. Environ. Qual. 2018, 47, 452-461. [CrossRef] [PubMed] 